LPT-Orsay-06-04

ITEP-TH-02/06

hep-th/0601112

\title{
Logarithmic scaling in gauge/string correspondence
}

\author{
A.V. Belitsky ${ }^{a}$, A.S. Gorsky ${ }^{b}$, G.P. KorchemskY ${ }^{c}$ \\ ${ }^{a}$ Department of Physics and Astronomy, Arizona State University \\ Tempe, AZ 85287-1504, USA \\ ${ }^{b}$ Institute of Theoretical and Experimental Physics \\ B. Cheremushkinskaya ul. 25, 117259 Moscow, Russia \\ ${ }^{c}$ Laboratoire de Physique Théorique ${ }^{1}$, Université de Paris XI \\ 91405 Orsay Cédex, France
}

\begin{abstract}
We study anomalous dimensions of (super)conformal Wilson operators at weak and strong coupling making use of the integrability symmetry on both sides of the gauge/string correspondence and elucidate the origin of their single-logarithmic behavior for long operators/strings in the limit of large Lorentz spin. On the gauge theory side, we apply the method of the Baxter $Q$-operator to identify different scaling regimes in the anomalous dimensions in integrable sectors of (supersymmetric) Yang-Mills theory to one-loop order and determine the values of the Lorentz spin at which the logarithmic scaling sets in. We demonstrate that the conventional semiclassical approach based on the analysis of the distribution of Bethe roots breaks down in this domain. We work out an asymptotic expression for the anomalous dimensions which is valid throughout the entire region of variation of the Lorentz spin. On the string theory side, the logarithmic scaling occurs when two most distant points of the folded spinning string approach the boundary of the AdS space. In terms of the spectral curve for the classical string sigma model, the same configuration is described by an elliptic curve with two branching points approaching values determined by the square root of the 't Hooft coupling constant. As a result, the anomalous dimensions cease to obey the BMN scaling and scale logarithmically with the Lorentz spin.
\end{abstract}

\footnotetext{
${ }^{1}$ Unité Mixte de Recherche du CNRS (UMR 8627).
} 


\section{Introduction}

It is well known that in four-dimensional gauge theories the anomalous dimensions of composite Wilson operators carrying a large Lorentz spin scale (at most) logarithmically with the spin. This result is just one of the facets of a more general Sudakov phenomenon [1] and it can be traced back to the existence of massless particles of spin one in the spectrum - the gauge fields. The logarithmic scaling of anomalous dimensions is a universal feature of all gauge theories ranging from QCD to the maximally supersymmetric $\mathcal{N}=4$ Yang-Mills (SYM) theory. In particular, in the simplest case of twist-two Wilson operators with large Lorentz spin $N \gg 1$, the anomalous dimension behaves as (in the adjoint representation of the $S U\left(N_{c}\right)$ group) [2]

$$
\gamma(\lambda)=2 \Gamma_{\text {cusp }}(\lambda) \ln N+\mathcal{O}\left(N^{0}\right)
$$

where $\lambda=g_{\mathrm{YM}}^{2} N_{c}$ is the 't Hooft coupling constant and $\Gamma_{\text {cusp }}(\lambda)$ is the so-called cusp anomalous dimension [3]. $\Gamma_{\text {cusp }}(\lambda)$ is not universal however and depends on the theory under consideration. It has numerous applications in phenomenology of strong interactions and its calculation both at weak and strong coupling regimes is one of the long-standing problems in gauge theories. At present, the cusp anomaly is known in perturbation theory to the lowest three orders 4, 5] and there exists a prediction at strong coupling in the $\mathcal{N}=4 \mathrm{SYM}$ theory based on the gauge/string correspondence [6, 7, 8].

The gauge/string correspondence [9] offers a powerful tool to study the dynamics of fourdimensional Yang-Mills theories at strong coupling. It establishes a correspondence between Wilson operators in $\mathcal{N}=4 \mathrm{SYM}$ theory and certain string excitations on the $\operatorname{AdS}_{5} \times \mathrm{S}^{5}$ background [10, 6]. For operators carrying large quantum numbers (Lorentz spin, isotopic $R$-charge,...) their scaling dimension at strong coupling can be found as an energy of dual (semi)classical string configurations propagating on the curved space. As was shown in Ref. [6], the operators of twist two with large Lorentz spin $N$ are dual to a folded string rotating with the angular momentum $N$ on the $\mathrm{AdS}_{3}$ part of the target space. The resulting expression for the twist-two anomalous dimension takes the form (1.1) with the cusp anomalous dimension at strong coupling given by

$$
\Gamma_{\text {cusp }}(\lambda) \stackrel{\lambda \gg 1}{=} \frac{\sqrt{\lambda}}{2 \pi}+\mathcal{O}\left((\sqrt{\lambda})^{0}\right) .
$$

Later, the dual string picture was generalized to Wilson operators of higher twist in the $\mathcal{N}=4$ SYM theory carrying both large Lorentz spin and the $R$-charge [11. For operators built from holomorphic scalar fields carrying a unit isotopic charge, the total $R$-charge equals the twist, $L$. For such operators, in the dual picture the center-of-mass of the string rotates with the angular momentum $L$ along a large circle of $\mathrm{S}^{5}$.

The important difference between the operators of twist two, $L=2$, and of higher twist, $L \geq 3$, is that the latter are not uniquely specified by the total Lorentz spin $N$. More precisely, for $L \geq 3$ there exist several (superconformal) operators with the same $N$. These operators mix under renormalization and the size of the mixing matrix rapidly increases with $L$ and $N$. As a consequence, the anomalous dimensions of Wilson operators of high twist $L \geq 3$ also depend on the integers $\ell=1,2, \ldots$ which enumerate eigenvalues of the mixing matrix (=anomalous dimensions) for a given Lorentz spin $N$. For fixed $L$ and large $N$, possible values of the anomalous dimension occupy a band [8]. The lower boundary of the band scales for $N \rightarrow \infty$ as in (1.1) while the upper boundary scales as $\sim L \Gamma_{\text {cusp }}(\lambda) \ln N$. This implies that for operators of twist 
$L=3,4, \ldots$ the minimal anomalous dimension has the same leading asymptotic behavior for $N \rightarrow \infty$ as the twist-two anomalous dimension, Eq. (1.1). This result is rather general and it holds true in a generic Yang-Mills theory including QCD and $\mathcal{N}=4$ SYM theory.

In the present paper, we study the properties of the minimal anomalous dimensions on both sides of the gauge/string correspondence in the limit of large twist $L$ and Lorentz spin $N$. On the string side, the corresponding single-trace Wilson operators are dual to a folded string spinning with large angular momentum $N$ in the $\mathrm{AdS}_{3}$ part of the anti-de Sitter space and boosted along a large circle on the sphere with a large angular momentum $L$ [1]. The energy $E$ of this classical string configuration defines the leading asymptotics of the anomalous dimension $\gamma(\lambda)=E-L-N$ of the dual Wilson operator in the $\mathcal{N}=4$ SYM theory in the strong coupling regime in planar approximation. The string theory provides a definite prediction for $\gamma(\lambda)$ as a function of $L$ and $N$. One finds that $\gamma(\lambda)$ takes different forms in three regimes [6, 11]:

- For $N \ll L$, in the "short" string limit

$$
\gamma(\lambda)=\lambda \frac{m^{2}}{2} \frac{N}{L^{2}}+\ldots
$$

- For $N \gg L$, in the "long" string limit

$$
\gamma(\lambda)= \begin{cases}\frac{\lambda}{2 \pi^{2}} \frac{m^{2}}{L} \ln ^{2}(N / L)+\ldots, & \text { for } \xi_{\mathrm{str}}<1, \\ \frac{\sqrt{\lambda}}{\pi} m \ln (N / \sqrt{\lambda})+\ldots, & \text { for } \xi_{\mathrm{str}} \gg 1,\end{cases}
$$

with the parameter $\xi_{\text {str }}$ defined as $\xi_{\text {str }}=\lambda \ln ^{2}(N / L) / L^{2}$. Here the integer $m$ counts the number of times the string is folded onto itself. The minimal anomalous dimension corresponds to a single-folded string, $m=1$. In that case, for $\xi_{\text {str }} \gg 1$, the leading asymptotic behavior of $\gamma(\lambda)$ does not depend on the twist $L$ and is the same as for $L=2$ operators, Eqs. (1.1) and (1.2). For $\xi_{\text {str }}<1$, the role of the twist $L$ is to create the "BMN window", i.e., a region in the parameter space in which the anomalous dimension has an expansion in powers of the BMN coupling $\lambda^{\prime} \equiv \lambda /(\pi L)^{2}$ [10]. It is believed that the first few terms in the expansion of $\gamma(\lambda)$ in powers of $\lambda^{\prime}$ should match similar expressions for the anomalous dimensions of Wilson operators of twist $L$ and spin $N$ obtained in the $\mathcal{N}=4$ SYM theory in the weak coupling regime [12].

On the gauge theory side, the calculation of anomalous dimensions of higher twist operators with large Lorentz spin turns out to be an extremely nontrivial task in a generic Yang-Mills theory even to one-loop order due to a large size of the mixing matrix. The problem can be overcome thanks to hidden integrability symmetry of the dilatation operator [13, 14, 15], which maps the one-loop mixing matrix for Wilson operators of twist $L$ belonging to the so-called holomorphic $S L(2)$ sector into a Hamiltonian of the Heisenberg magnet of length $L$ and spin $s$ determined by the conformal spin of the quantum fields (for a review see Ref. [16]). This observation allows one to calculate the exact eigenspectrum of anomalous dimensions of Wilson operators of arbitrary twist and Lorentz spin in integrable sectors of Yang-Mills theory by means of the Quantum Inverse Scattering Method [17]. In the $\mathcal{N}=4 \mathrm{SYM}$ theory, the minimal anomalous dimension of Wilson operators built from $L$ scalar fields and carrying the Lorentz spin $N$ can be identified to one-loop accuracy as the minimal energy in the eigenspectrum of the $S L(2)$ Heisenberg magnet of length $L$ and the total spin $N+L s$ with $s=\frac{1}{2}[18$. The gauge/string correspondence suggests 
that the minimal anomalous dimension defined in this way should match the relations (1.3) and (1.4) in the thermodynamic limit $L \rightarrow \infty$.

It follows from (1.3) and (1.4) that the anomalous dimensions of higher twist operator depend on a "hidden" parameter $\xi_{\text {str }}=\lambda \ln ^{2}(N / L) / L^{2}$ and their behavior at strong coupling is different for $\xi_{\text {str }}<1$ and $\xi_{\text {str }} \gg 1$. For $\xi_{\text {str }} \gg 1$ the anomalous dimension does not have a perturbative expansion in the BMN coupling $\lambda^{\prime}$ and scales as $\sim \ln N$. On the gauge theory side, previous studies [18] of the Bethe ansatz for the $S L(2)$ spin chain in the thermodynamic limit $L \gg 1$ led to the expression for $\gamma(\lambda)$ which coincides with (1.3) in the limit of short strings and with the first relation in (1.4) in the limit of long strings. They did not reveal however neither any trace of the second, logarithmic regime in (1.4), nor appearance of a new parameter similar to $\xi_{\text {str }}$. This fact is in contradiction with our expectations that the minimal anomalous dimension of higher twist operators should scale logarithmically to all loops as $N \rightarrow \infty$, Eq. (1.1). The goal of the present study is to unravel the logarithmic scaling of the anomalous dimension both in the gauge and string theory and to understand the physical meaning of the parameter $\xi_{\text {str }}$ and its counter-part $\xi$ on the gauge theory side.

We shall revisit the calculation of the energy of the $S L(2)$ Heisenberg magnet of $\operatorname{spin} s=\frac{1}{2}, 1, \frac{3}{2}$ using the method of the Baxter $Q$-operator [19] as a main tool and demonstrate that the oneloop anomalous dimension in integrable sectors of Yang-Mills theory has the following scaling behavior in the thermodynamic limit $L \rightarrow \infty$ :

- For $N \ll L$

$$
\gamma(\lambda)=\lambda \frac{m^{2}}{4 s} \frac{N}{L^{2}}+\ldots
$$

- For $N \gg L$

$$
\gamma(\lambda)= \begin{cases}\frac{\lambda}{2 \pi^{2}} \frac{m^{2}}{L} \ln ^{2}(N / L)+\ldots, & \text { for } \xi<1, \\ \frac{\lambda}{2 \pi^{2}} m \ln N+\ldots, & \text { for } \xi \gg 1,\end{cases}
$$

depending on the parameter $\xi=\ln (N / L) / L$. The minimal anomalous dimension corresponds to $m=1$. Here $s$ equals the conformal spin of the field entering the Wilson operator, i.e., $s=\frac{1}{2}, 1, \frac{3}{2}$ for scalar, gaugino and gluon fields, respectively. For scalar operators, Eq. (1.5) and the first relation in (1.6) coincide with similar expressions in (1.3) and (1.4), respectively. Notice that the anomalous dimension in (1.6) does not depend on the spin $s$ for $N \gg L$ which suggests that the two regimes in (1.6) are universal in all gauge theories. This is indeed the case for $\xi \gg 1$ since the coefficient in front of $2 \ln N$ coincides with the cusp anomalous dimension at weak coupling, Eq. (1.1).

For $N \gg L$ and $\xi<1$, the one-loop anomalous dimension exhibits a novel double logarithmic behavior (1.6). It was first discovered from the string theory considerations [1] and was later reproduced on the gauge theory side [18. A natural question arises whether similar contributions arise at higher loops and whether they can be resummed to all loops. The gauge/string correspondence suggests that in the $\mathcal{N}=4 \mathrm{SYM}$ theory the anomalous dimension in the region $N \gg L \gg 1$ admits a BMN-like expansion (with $\lambda^{\prime}=\lambda /(\pi L)^{2}$ and $\xi=\ln (N / L) / L$ )

$$
\gamma(\lambda)=L \sum_{n=1}^{\infty}\left(\lambda^{\prime} \ln ^{2} \frac{N}{L}\right)^{n} c_{n}(\xi)+\ldots
$$


where the coefficient functions $c_{n}(\xi)$ do not depend on the coupling constant and have the following asymptotics for $\xi \rightarrow 0$ and $\xi \rightarrow \infty$

$$
c_{n}(\xi)=c_{0, n}+c_{1, n} \xi+\mathcal{O}\left(\xi^{2}\right), \quad c_{n}(\xi)=\mathcal{O}\left(1 / \xi^{2 n-1}\right)
$$

with $c_{0, n}=(-1)^{n}\left(-\frac{1}{2}\right)_{n} / n$ !. To one-loop order, Eq. (1.7) matches (for $m=1$ ) both relations in (1.6). In addition, for $\xi \rightarrow \infty$ the coefficient in front of $\lambda^{n}$ in the right-hand side of (1.7) scales as $\sim \ln N$ and determines the $n$-loop correction to the cusp anomalous dimension in the weak coupling regime, Eq. (1.1). In the strong coupling regime, upon the substitution $\xi=\xi_{\text {str }}^{1 / 2} / \sqrt{\lambda}$, the perturbative series in (1.7) can be resummed to all loops into the following expression (for $\lambda \rightarrow \infty$ and $\xi_{\text {str }}=\lambda \ln ^{2}(N / L) / L^{2}=$ fixed $)$

$$
\gamma(\lambda)=L \sum_{n=1}^{\infty}\left(\lambda^{\prime} \ln ^{2} \frac{N}{L}\right)^{n} c_{n}(0)+\ldots=L\left[\sqrt{1+\lambda^{\prime} \ln ^{2} \frac{N}{L}}-1\right]+\ldots
$$

where the ellipsis stands for subleading corrections. For $N \rightarrow \infty$ this relation also reproduces the leading asymptotic behavior of the anomalous dimension in the last regime in (1.4) and, as a consequence, it leads to the expression for the cusp anomalous dimension at strong coupling, Eq. (1.2).

As was already mentioned, the one-loop anomalous dimension (1.7) coincides with the energy of the $S L(2)$ spin chain of length $L$ in the thermodynamic limit $L \rightarrow \infty$. The latter can be found within the Bethe Ansatz approach by systematically expanding the energy in powers of $1 / L$ with a help of known semiclassical methods 20. To leading order of the semiclassical expansion, the Bethe roots condense on two symmetric intervals on the real axis $[-a,-b] \cup[b, a]$, with the boundaries $a$ and $b$ being functions of $L / N$. This leads [18] to the expression for the energy given in (1.3) for $N \ll L$ and in the first relation in (1.4) for $N \gg L$. It is believed that subleading corrections to the energy are suppressed by powers of $1 / L$ and, therefore, are small. We demonstrate that this assumption is only justified for $\xi=\ln (N / L) / L<1$, while for $\xi \gg 1$ the semiclassical expansion of the energy becomes divergent indicating the change of asymptotic behavior of the anomalous dimension, Eq. (1.4). The reason why the semiclassical expansion fails is that the two cuts $[-a,-b]$ and $[b, a]$ collide at the origin, that is $b \rightarrow 0$ for $\xi \rightarrow \infty$, and the Bethe roots have a nonvanishing distribution at the origin. As a consequence, for $N \gg L$ the semiclassical corrections to the anomalous dimension run in powers of $\xi$. To one-loop order they are described in (1.7) by the function $c_{1}(\xi)$. We argue that the semiclassical series for $c_{1}(\xi)$ is divergent for $\xi>1$ and propose an approach which circumvents this difficulty and allows one to determine this function for arbitrary $\xi$. The resulting expression for the one-loop anomalous dimension is valid in the thermodynamic limit throughout the entire interval of $N$ and reproduces correct logarithmic behavior for $N \gg L$, Eq. (1.6).

On the string theory side, in the dual picture of the folded string spinning in the $\mathrm{AdS}_{3} \times \mathrm{S}^{1}$ part of the target space, the logarithmic behavior of the anomalous dimension at strong coupling is associated with the classical string configuration which has two spikes approaching the boundary of the AdS space. Thanks to classical integrability of the string equations of motion [21], the same configuration is described by the spectral (elliptic) symmetric curve endowed with a meromorphic differential of quasimomentum possessing a double pole at $x= \pm \sqrt{\lambda^{\prime}}$ and having a prescribed asymptotic behavior at the origin and infinity [22, 23, 24]. The branching points of the curve, $\pm b_{\text {str }}$ and $\pm a_{\text {str }}$, depend on the ratio $L / N$ and the coupling constant $\lambda^{\prime}$. We show that for $N \gg L$ and $\xi_{\text {str }}<1$ the branching points admit a regular expansion in powers of $\lambda^{\prime}$ and, as 
a consequence, the anomalous dimension exhibits the BMN scaling, Eq. (1.4). For $\xi_{\text {str }} \gg 1$, $b_{\text {str }}$ approaches its minimal value $\sqrt{\lambda^{\prime}}$ so that the inner boundaries of two cuts $\left[-a_{\text {str }},-b_{\text {str }}\right]$ and $\left[b_{\text {str }}, a_{\text {str }}\right]$ coincide with the position of poles of the momentum differential and cannot collide. This nonanalyticity manifests itself through the appearance of $\sqrt{\lambda}$ prefactor in the logarithmic behavior of the anomalous dimension at strong coupling, Eq. (1.4).

Our consequent presentation is organized as follows. In Sect. 2, we outline a general framework for analysis of one-loop anomalous dimensions in the thermodynamic limit. It is based on the semiclassical expansion of solutions to the Baxter equation [25, 26, 27]. In Sect. 3, we apply the semiclassical approach to determine the minimal anomalous dimension in the thermodynamic limit and demonstrate that the semiclassical expansion breaks down for $\ln (N / L) \gg L$ due to collision of cuts. Then, we present an approach to go consistently beyond the semiclassical expansion and use it to describe the minimal anomalous dimension for large Lorentz spin. In Sect. 15. we analyze the asymptotic behavior of the anomalous dimensions at strong coupling based on the string sigma model consideration. Section 4 contains concluding remarks. Some technical details of our calculations are summarized in the Appendix.

\section{Anomalous dimensions in gauge theory}

Let us start with the calculation of one-loop anomalous dimensions of (super)conformal Wilson operators of arbitrary twist $L$ and Lorentz spin $N$ belonging to integrable sectors of (supersymmetric) Yang-Mills theories. For quantum fields transforming in the adjoint representation of the gauge group, the operators under considerations have the following generic form

$$
\mathcal{O}_{N, L}(0)=\sum_{k_{1}+\ldots+k_{L}=N} c_{k_{1} \ldots k_{L}} \operatorname{tr}\left\{D_{+}^{k_{1}} X(0) D_{+}^{k_{2}} X(0) \ldots D_{+}^{k_{L}} X(0)\right\}
$$

where $X(0)$ stands for the so-called "good" component of quantum fields of a definite helicity in the underlying gauge theory, $D_{+}=D_{\mu} n^{\mu}$ is the covariant derivative projected onto the light-cone,

$n_{\mu}^{2}=0$. The expansion coefficients $c_{k_{1} \ldots k_{L}}$ are fixed from the condition for $\mathcal{O}_{N, L}(0)$ to have an autonomous scale dependence, i.e., Eq. (2.1) has to be an eigenstate of the one-loop dilatation operator. Integrability allows one to map the one-loop anomalous dimension of the operators (2.1) into energy $\varepsilon$ of the noncompact $S L(2)$ Heisenberg spin chain of length $L$ and the total spin $N+L s$ [13, 14, 15, 28,

$$
\gamma(\lambda)=\frac{\lambda}{8 \pi^{2}} \varepsilon+\mathcal{O}\left(\lambda^{2}\right) .
$$

Here the (half-)integer $s$ is given by the conformal spin [16] of the quantum field $X(0)$, that is, $s=1 / 2$ for scalars, $s=1$ for gaugino fields of helicity $\pm 1 / 2$ and $s=3 / 2$ for gauge fields of helicity \pm 1 .

\subsection{Exact solution}

Let us first describe the exact solution for the energy $\varepsilon$ of the $S L(2)$ magnet of length $L$ and single-particle spin $s$ in each site. We shall employ the method of the Baxter $Q$-operator [19] which proves to be convenient for analyzing various semiclassical limits of $\varepsilon=\varepsilon(N, L)$ including the limit of the large spin $N$ and length $L$ [26, 27]. The method relies on the existence of an operator $Q(u)$ which acts on the Hilbert space of the $S L(2)$ spin chain and is diagonalized by all 
eigenstates of the magnet for arbitrary complex parameter $u$. Discussing the energy spectrum it suffices to study the eigenvalues of the $Q$-operator that we shall denote by $Q(u)$. The same function $Q(u)$ determines the wave function of the magnet in the representation of Separated Variables [29] ${ }^{1}$. This allows one to analyze $Q(u)$ in the semiclassical limit with the help of the WKB machinery well-known from quantum mechanics and, then, determine $\varepsilon(N, L)$.

By construction, $Q(u)$ satisfies the second-order finite-difference equation

$$
(u+i s)^{L} Q(u+i)+(u-i s)^{L} Q(u-i)=t_{L}(u) Q(u),
$$

which can be thought of as a Schrödinger equation for a single-particle wave function in the representation of Separated Variables [29. Here $t_{L}(u)$ is a polynomial in $u$ of degree $L$ with coefficients given by conserved charges

$$
t_{L}(u)=2 u^{L}+q_{2} u^{L-2}+\ldots+q_{L}
$$

The lowest integral of motion $q_{2}$ is related to the total spin of the $S L(2)$ chain, $N+L s$,

$$
q_{2}=-(N+L s)(N+L s-1)+L s(s-1)
$$

with $N=0,1, \ldots$

In what follows we shall refer to Eq. (2.3) as the Baxter equation. Taken alone, it does not fix the function $Q(u)$ and it has to be supplemented by an additional condition that $Q(u)$ has to be polynomial in $u$ 31. Examining the asymptotic behavior of both sides of (2.3) for $u \rightarrow \infty$, it is easy to see that the degree of $Q(u)$ is fixed by the total spin $N$ and, therefore, up to an overall normalization, one can write

$$
Q(u)=\prod_{k=1}^{N}\left(u-\lambda_{k}\right) .
$$

One substitutes this ansatz into (2.3), takes $u=\lambda_{k}$ and finds that the roots $\lambda_{1}, \ldots, \lambda_{N}$ satisfy the Bethe equations

$$
\left(\frac{\lambda_{k}+i s}{\lambda_{k}-i s}\right)^{L}=\prod_{j=1, j \neq k}^{N} \frac{\lambda_{k}-\lambda_{j}-i}{\lambda_{k}-\lambda_{j}+i} .
$$

Solving the Baxter equation (2.3) supplemented by (2.6) one obtains quantized values of the charges $q_{3}, \ldots, q_{L}$ and evaluates the corresponding energy and quasimomentum as 31]

$$
\varepsilon=i(\ln Q(i s))^{\prime}-i(\ln Q(-i s))^{\prime}, \quad \mathrm{e}^{i \theta}=\frac{Q(i s)}{Q(-i s)} .
$$

Replacing $Q(u)$ by its expression (2.6) one verifies that these relations coincide with those coming from the Algebraic Bethe Ansatz [17]

$$
\varepsilon=\sum_{k=1}^{N} \frac{2 s}{\lambda_{k}^{2}+s^{2}}, \quad \mathrm{e}^{i \theta}=\prod_{k=1}^{N} \frac{\lambda_{k}-i s}{\lambda_{k}+i s} .
$$

The cyclic symmetry of the single-trace operators (2.1) imposes an additional selection rule for the eigenstates of the spin magnet, $\mathrm{e}^{i \theta}=1$. Equations (2.8) and (2.9) allow one to calculate the energy of the spin chain and, then, obtain the one-loop anomalous dimension of Wilson operators (2.1) with a help of (2.2).

\footnotetext{
${ }^{1}$ For an interpretation of the $Q$-operator in string theory see Ref. 30].
} 


\subsection{Quasiclassical approach}

Let us examine the Baxter equation (2.3) for $N+L s \gg 1$. In this limit, the charge $q_{2}$ takes large negative values and one can apply semiclassical techniques [26] to construct the solution to (2.3). To go over to the semiclassical limit, we introduce two scaling parameters

$$
\eta=(N+L s)^{-1}, \quad \beta=s L \eta=\frac{s L}{N+L s} .
$$

By definition, $0 \leq \beta \leq 1$ with the boundary values corresponding to

$$
\beta \stackrel{L \ll N}{\longrightarrow} 0, \quad \beta \stackrel{L \gg N}{\longrightarrow} 1 .
$$

The parameter $\eta \ll 1$ will play the role of the Planck constant. One rescales the spectral parameter as $u=x / \eta$ and introduces the eikonal phase (the Hamilton-Jacobi "action" function) $S(x)$ as

$$
Q(x / \eta)=\eta^{-N} \exp \left(\frac{1}{\eta} S(x)\right), \quad S(x)=\eta \sum_{k=1}^{N} \ln \left(x-\eta \lambda_{k}\right) .
$$

The energy and the quasimomentum in Eq. (2.8) are then given in terms of the function $S(x)$ by the following expressions

$$
\varepsilon=i\left[S^{\prime}(i \beta / L)-S^{\prime}(-i \beta / L)\right], \quad \mathrm{e}^{i \theta}=\exp \left\{\frac{1}{\eta}[S(i \beta / L)-S(-i \beta / L)]\right\} .
$$

It also proves convenient to introduce a notation for the "effective potential"

$$
\tau(x)=(\eta / x)^{L} t_{L}(x / \eta)=2+\frac{\widehat{q}_{2}}{x^{2}}+\frac{\widehat{q}_{3}}{x^{3}}+\ldots+\frac{\widehat{q}_{L}}{x^{L}}
$$

with $\widehat{q}_{k}=q_{k} \eta^{k}$.

In the semiclassical approach [25, 26] one assumes that the function $S(x)$ and the integrals of motion $q_{k}$ (with $\left.k=3, \ldots, L\right)$ admit a systematic expansion in powers of $\eta$

$$
S(x)=S_{0}(x)+\eta S_{1}(x)+\ldots, \quad \widehat{q}_{k}=\widehat{q}_{k}^{(0)}+\eta \widehat{q}_{k}^{(1)}+\ldots .
$$

It is tacitly assumed that the expansion of $S(x)$ is convergent and each term is uniformly bounded. ${ }^{2}$ This leads to the expansion of the effective potential (2.14), $\tau(x)=\tau_{0}(x)+\eta \tau_{1}(x)+\ldots$ with

$$
\tau_{0}(x)=2-\frac{1}{x^{2}}+\frac{\widehat{q}_{3}^{(0)}}{x^{3}}+\ldots+\frac{\widehat{q}_{L}^{(0)}}{x^{L}}, \quad \tau_{1}(x)=\frac{\widehat{q}_{3}^{(1)}}{x^{3}}+\ldots+\frac{\widehat{q}_{L}^{(1)}}{x^{L}}, \quad \ldots
$$

One substitutes (2.15) into the Baxter equation (2.3) and equates the coefficients in front of powers of $\eta$ to get to leading order

$$
2 \cos p(x)=\tau_{0}(x), \quad p(x)=S_{0}^{\prime}(x)+\frac{\beta}{x} .
$$

In the finite-gap theory, the function $p(x)$ defines the Bloch-Floquet multiplier in an auxiliary linear problem for the Baker-Akhiezer function and has the meaning of the (quasi)momentum

\footnotetext{
${ }^{2}$ As we will show in Sect. [3] this assumption is justified for $\ln (N / L)<L$ and is invalid otherwise.
} 
32, 33, 34. For the first subleading term in the semiclassical expansion one finds in a similar manner

$$
S_{1}^{\prime}(x)=-\frac{p^{\prime}(x)}{2} \cot p(x)-\frac{1}{2 \sin p(x)}\left(\tau_{1}(x)+\frac{\beta(1-s)}{2 x^{2}} \tau_{0}(x)\right) .
$$

It is straightforward to derive the subleading terms $S_{k \geq 2}^{\prime}(x)$ but we will not need them for our purposes. The obtained expressions for the action functions $S_{0}^{\prime}(x)$ and $S_{1}^{\prime}(x)$ depend on yet unknown conserved charges $\widehat{q}_{k}^{(0)}$ and $\widehat{q}_{k}^{(1)}$, respectively. Quantization conditions for these charges follow from the requirement for $Q(x / \eta)$, Eq. (2.12), to be a single valued function of $x$.

According to (2.16), $\tau_{0}(x)$ is a polynomial of degree $L$ in $1 / x$. Solving (2.17), one finds that the momentum $p(x)$ is, in general, a double-valued function on the complex $x$-plane with the square-root branching points $x_{j}$ obeying the condition $\tau_{0}\left(x_{j}\right)= \pm 2$. It is convenient to introduce the function $y(x)=2 \sin p(x)$ and define a complex curve [26]

$$
\Gamma_{L}: \quad y^{2}=4-\tau_{0}^{2}(x), \quad \tau_{0}(x)=2-\frac{1}{x^{2}}+\frac{\widehat{q}_{3}^{(0)}}{x^{3}}+\ldots+\frac{\widehat{q}_{L}^{(0)}}{x^{L}} .
$$

For arbitrary complex $x$, except the branching points $y\left(x_{j}\right)=0$, the relation (2.19) defines two values for $y(x)$. Then, $y(x)$ being a double-valued function on the complex $x$-plane, becomes a single-valued function on the hyperelliptic Riemann surface defined by the complex curve $\Gamma_{L}$. This surface has a genus $L-2$ and is realized by gluing together two copies of the complex $x$-plane along the cuts running between the branching points $x_{2 j-1}$ and $x_{2 j}$.

For the $S L(2)$ magnet the Bethe roots verifying Eq. (2.7) take real values only, $\operatorname{Im} \lambda_{k}=0$. In the semiclassical limit, $\eta \rightarrow 0$, they condense on finite intervals on the real axis where the momentum $p(x)$ takes purely imaginary values [26]. In terms of the hyperelliptic curve (2.19), this corresponds to $y^{2} \leq 0$, or

$$
\tau_{0}^{2}(x) \geq 4, \quad \text { for } x \in \mathcal{S}=\left[x_{2 L-2}, x_{2 L-3}\right] \cup \ldots \cup\left[x_{4}, x_{3}\right] \cup\left[x_{2}, x_{1}\right],
$$

where $x_{1}>x_{2}>\ldots>x_{2 L-2}$ and one of the intervals contains the origin. The total number of intervals in (2.20) equals $L-1$ and the end points $x_{j}$ are just the branching points of the complex curve (2.19), $\tau_{0}^{2}\left(x_{j}\right)=4$. As follows from (2.19), the curve can be parameterized by the set of $2 L-2$ real branching points as

$$
y^{2}=\frac{4}{x^{2}} \prod_{j=1}^{2 L-2}\left(1-\frac{x_{j}}{x}\right) .
$$

The intervals $\mathcal{S}$ have the meaning of regions where the classical motion of the system occurs in the separated variables ${ }^{3}$. Later on we shall encounter the situation when, say, $j^{\text {th }}$ interval shrinks into a point, $x_{2 j}=x_{2 j-1}$, so that the motion on this interval is frozen at the classical level. In what follows we shall refer to $x_{2 j}=x_{2 j-1}$ as a double point.

The leading term of the semiclassical expansion, $S_{0}(x)$, is determined by the momentum $p(x)$, Eq. (2.17). As follows from its definition (2.17)

$$
p(x)=2 i \ln \frac{\sqrt{\tau_{0}(x)+2}-\sqrt{\tau_{0}(x)-2}}{2} .
$$

\footnotetext{
${ }^{3}$ In the finite-gap theory $[32$, the same intervals have the meaning of forbidden zones in the auxiliary linear problem for the Baker-Akhiezer function.
} 
The momentum $p(x)$ takes purely imaginary values on the intervals (2.20) and its values at the end point of the $j^{\text {th }}$ interval coincide, $p\left(x_{2 j-1}\right)=p\left(x_{2 j}\right)$, with $\mathrm{e}^{i p\left(x_{2 j}\right)}= \pm 1$ for $\tau_{0}\left(x_{2 j}\right)= \pm 2$, respectively. Continuing $p(x)$ to the complex $x$-plane one finds that $p^{\prime}(x)$ is an analytical function on the complex plane with cuts running along the intervals (2.20). It defines a meromorphic differential on $\Gamma_{L}$

$$
d p=p^{\prime}(x) d x=-\frac{\tau_{0}^{\prime}(x)}{\sqrt{4-\tau_{0}^{2}(x)}} d x .
$$

From (2.19) and (2.22) one finds $\tau_{0}(x)=2-1 / x^{2}+\mathcal{O}\left(1 / x^{3}\right)$ so that $\mathrm{e}^{p(\infty)}=1$ and

$$
d p \sim \mp \frac{d x}{x^{2}}, \quad \text { for } x \rightarrow \infty
$$

where ' $-/+$ ' correspond to the upper/lower sheet of $\Gamma_{L}$. According to the definition (2.17), $p(\infty)$ is defined modulo $2 \pi$. Choosing the normalization condition $p(\infty)=0$, one finds that at the end points of the $j^{\text {th }}$ interval in (2.20), the momentum takes the values $p\left(x_{2 j}\right)=p\left(x_{2 j-1}\right)=-\pi j$ for $x_{2 j}>0$. As a consequence, the differential $d p$ satisfies the normalization conditions [35, 32]

$$
2 \int_{x_{2 j}}^{x_{2 j-1}} d x p^{\prime}(x)=-\oint_{\alpha_{j}} d p=0, \quad 2 \int_{x_{2 j-1}}^{\infty} d x p^{\prime}(x)=-\int_{\gamma_{j}} d p=-2 \pi j .
$$

Here in both equations, the integration in the left-hand side goes over the upper sheet of $\Gamma_{L}$. In the right-hand side of the first relation, the differential $d p$ is integrated over the $\alpha_{j}-$ cycle encircling the interval $\left[x_{2 j}, x_{2 j-1}\right]$ in the anticlockwise direction. The contour $\gamma_{j}$ in the second relation starts on the upper sheet above $x=\infty$ crosses the same interval and then goes to infinity on the lower sheet (see Fig. 11).

The obtained expressions for $S_{0}^{\prime}(x)$ and $S_{1}^{\prime}(x)$, Eqs. (2.17) and (2.18), respectively, depend on the conserved charges $\widehat{q}_{k}$, yet to be determined. To work out the quantization conditions for $\widehat{q}_{k}$ one examines the first derivative of the eikonal phase (2.12)

$$
S^{\prime}(x)=\eta \sum_{k=1}^{N} \frac{1}{x-\eta \lambda_{k}} .
$$

The discontinuity of $S^{\prime}(x)$ across the cuts (2.20) gives the distribution density of rescaled Bethe roots $\eta \lambda_{k}$. Assuming that the roots $\eta \lambda_{k}$ take finite values for $\eta \rightarrow 0$, one finds the behavior of $S^{\prime}(x)$ at infinity on the upper, physical sheet of $\Gamma_{L}$ as

$$
S^{\prime}(x) \sim \frac{\eta N}{x}=\frac{1-\beta}{x}, \quad \text { for } x \rightarrow \infty,
$$

with $\eta$ and $\beta$ defined in (2.10). Replacing $S(x)$ by its semiclassical expansion (2.15) and matching the coefficients in front of powers of $\eta$ one obtains ${ }^{4}$

$$
S_{0}^{\prime}(x) \sim \frac{1-\beta}{x}, \quad S_{1}^{\prime}(x) \sim \frac{1}{x^{2}}, \quad \ldots
$$

According to (2.6), the total number of Bethe roots equals $N$. For a given energy level they

\footnotetext{
${ }^{4}$ Later on we shall consider solutions to the Baxter equation satisfying $Q(u)=Q(-u)$, or equivalently $S(x)=$ $S(-x)$. For such solutions, $S_{k}^{\prime}(x)$ are odd functions of $x$ and their asymptotics at infinity involves odd powers of $x$ only, that is, $S_{k \geq 1}^{\prime}(x) \sim 1 / x^{3}$.
} 


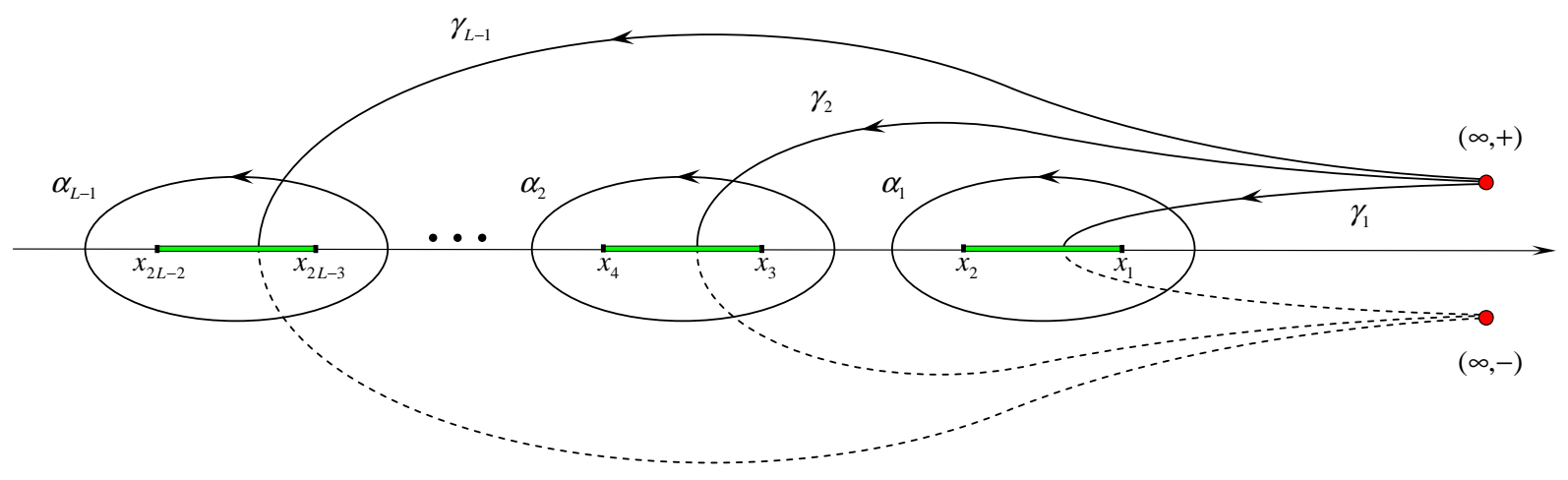

Figure 1: The definition of the $\alpha$-cycles and $\gamma$-contours on the Riemann surface $\Gamma_{L}$. The dashed lines represent the part of the path on the lower sheet of the surface.

are distributed on $L-1$ intervals (2.20). Denoting by $\ell_{j}$ the number of Bethe roots on the $j^{\text {th }}$ interval, one has

$$
\frac{1}{2 \pi i} \oint_{\alpha_{j}} d x S^{\prime}(x)=\eta \ell_{j}=\frac{\ell_{j}}{N+L s}, \quad(j=1, \ldots, L-1)
$$

The sum of all $\alpha$-cycles is homologous to zero and, as a consequence, the sum of all $\alpha$-periods is given by the residue of $S^{\prime}(x)$ at infinity. Together with (2.27) this leads to $N=\ell_{1}+\ldots+\ell_{L-1}$.

Solving the quantization conditions (2.29) one can determine quantized values of the conserved charges $\widehat{q}_{k}$. According to (2.29) they depend both on the scaling parameter $\eta$ and the set of nonnegative integers $\ell_{1}, \ldots, \ell_{L-1}$. Replacing $\widehat{q}_{k}$ in (2.16), (2.17) and (2.18) by their quantized values, one constructs semiclassical expression for $S(x)$ and, then, applies (2.13) to determine the energy and quasimomentum,

$$
\widehat{q}_{k}=\widehat{q}_{k}\left(\ell_{1}, \ldots, \ell_{L-1} ; \eta\right), \quad \varepsilon=\varepsilon\left(\ell_{1}, \ldots, \ell_{L-1} ; \eta\right) .
$$

Explicit form of these relations for $L=3$ can be found in Refs. 26, 13, 14, 15]. In particular, the quantized values of the energy and conserved charges exhibit remarkable regularity and form trajectories. The flow parameter along each trajectory is given by the total spin $\eta=$ $(N+L s)^{-1}$ while the integers $\ell_{1}, \ldots, \ell_{L-1}$ enumerate the trajectories and encode a nontrivial analytic structure in the eigenspectrum. For given $N$, the total number of trajectories equals the number of partitions of $N$ into the sum of $L-1$ nonnegative integers $\ell_{1}, \ldots, \ell_{L-1}$. This is in a perfect agreement with the number of irreducible components entering the tensor product of $L$ copies of the $S L(2)$ modules [8].

\subsection{Minimal energy trajectory}

In the semiclassical approach described in the previous section, the energy of the spin magnet, or equivalently the one-loop anomalous dimension of Wilson operators, is parameterized by the complex curve $\Gamma_{L}$, Eq. (2.19). The genus of the curve $g=L-2$ is defined by the length of the spin chain whereas its moduli are determined by the quantized values of the conserved charges $\widehat{q}_{k}$ which depend in their turn on the set of integers $\ell_{1}, \ldots, \ell_{L-1}$. Going over to different parts of the spectrum amounts to specifying the integers $\ell_{1}, \ldots, \ell_{L-1}$. 
In this paper we are interested in the eigenstates possessing the minimal possible energy for a given total spin $N$. Such eigenstates belong to a particular trajectory to which we shall refer as the minimal energy trajectory (see Fig. 4 below). To describe it one has first to identify the corresponding integers $\ell_{1}, \ldots, \ell_{L-1}$. We remind that the energy (2.9) is determined by zeros of the function $Q(u)$, or equivalently the Bethe roots. In the thermodynamical limit they condense on the intervals (2.20). For a given total spin $N$, the minimal energy is realized when the Bethe roots are located on two symmetric cuts most distant from the origin [20], that is, $\ell_{1}=\ell_{L-1}=$ $N / 2$ and $\ell_{j}=0$ for $j=2, \ldots, L-2$. The corresponding $Q(u)$ should be an even function, $Q(u)=Q(-u)$. According to (2.8), (2.6) and (2.3), such states automatically satisfy the cyclic symmetry condition $\mathrm{e}^{i \theta}=1$ and possess the following quantum numbers

$$
N=\text { even }, \quad q_{2 k+1}=0,
$$

with $0 \leq k \leq(L-1) / 2$. The fact that $\ell_{j}=0$ implies that $j^{\text {th }}$ interval does not contain Bethe roots and, therefore, it shrinks into a point, $x_{2 j-1}=x_{2 j}$. From the point of view of separated variables, this means that classically all but two collective degrees of freedom are frozen and the classical motion is confined to the two intervals with $\ell_{1}=\ell_{L-1}=N / 2$. For the complex curve (2.21), this implies that all branching points except four, $x_{1}=-x_{2 L-2}$ and $x_{2}=-x_{2 L-3}$, become the double points, $x_{2 j-1}=x_{2 j}$, and the curve $\Gamma_{L}$ reduces to the elliptic curve (see Eq. (2.37) below).

Let us apply the semiclassical approach to obtain the expression for the energy for $L \gg 1$ along the minimal energy trajectory as a function of $N$. To begin with, we consider the ground state of the $S L(2)$ spin chain. It has the total spin $N=0$ and is described by a trivial solution to the Baxter equation (2.3), $Q(u)=1$, or equivalently $S(x)=0$. From (2.8), the corresponding energy is $\varepsilon=0$ and the integrals of motion can be read off from (2.3) upon substituting $Q(u)=1$. To leading order in $\eta=1 /(s L)$ the transfer matrix and momentum, Eq. (2.17), look like

$$
\tau_{0}(x)=2 \cos (1 / x), \quad p(x)=1 / x .
$$

Matching these expressions into (2.19) and (2.21) one finds that for $L \rightarrow \infty$ all branching points of the spectral curve (2.21) are double points, $x_{2 j-1}=x_{2 j}=1 /(\pi j)$.

Let us now consider the minimal energy eigenstates with $N \gg 1$ and $L \gg 1$ and distinguish two limiting cases: (i) $N / L=$ fixed and (ii) $N / L \gg 1$, corresponding to $0<\beta<1$ and $\beta \rightarrow 0$, respectively, Eq. (2.10). We recall that the function $Q(u)$ has exactly $N$ roots, Eq. (2.6) . Assuming that the Bethe roots scale as $\lambda_{k} \sim 1 / \eta$, one finds from (2.9) that for $N / L=$ fixed the energy should behave as $\varepsilon \sim N / L^{2} \sim 1 / L$. Indeed, one can obtain the same scaling of $\varepsilon$ by naively expanding the energy (2.13) in powers of $1 / L$

$$
\varepsilon=-\frac{2 \beta}{L} S^{\prime \prime}(0)+\mathcal{O}\left(\beta^{3} / L^{3}\right)=-\frac{2 \beta}{L}\left[S_{0}^{\prime \prime}(0)+\eta S_{1}^{\prime \prime}(0)+\mathcal{O}\left(\eta^{2}\right)\right] .
$$

Here in the second relation we replaced $S(x)$ by its semiclassical expansion (2.15). In a similar manner, one obtains for the quasimomentum (2.13)

$$
\theta=2 s\left[S_{0}^{\prime}(0)+\eta S_{1}^{\prime}(0)+\mathcal{O}\left(\eta^{2}\right)\right]=0,
$$

where we took into account that $S_{k}(x)$ are even functions of $x$ for the minimal energy states

$$
S_{0}^{\prime}(0)=0, \quad S_{1}^{\prime}(0)=0, \quad \cdots
$$


Then, one finds from (2.17) the asymptotic behavior of the momentum around the origin on the upper ("physical") sheet of the Riemann surface (2.19) as

$$
p(x)=\frac{\beta}{x}+\mathcal{O}(x),
$$

so that the differential $d p$ has a double pole above $x=0$. It is important to stress that the relations (2.33) and (2.36) were obtained under the assumption that $S^{\prime}(x)$ is regular at the origin. For the minimal energy eigenstates, all but two intervals in (2.20) shrink into the double points and the Bethe roots condense on two symmetric cuts on the real axis that we shall denote as $[-a,-b]$ and $[b, a]$. Then, the spectral curve (2.21) reduces to

$$
y^{2}=\tilde{y}^{2}\left[\frac{2}{x^{3}} \prod_{j}\left(1-\frac{x_{2 j}^{2}}{x^{2}}\right)\right]^{2}, \quad \tilde{y}^{2}=\left(x^{2}-a^{2}\right)\left(x^{2}-b^{2}\right),
$$

where we took into account that for the minimal energy states the branching points appear in pairs of opposite sign. The parameters $a, b$ and $x_{2 j}$ are determined by the condition for $S_{0}^{\prime}(x)$, Eq. (2.17), to be an analytical function on the complex $x$-plane with two symmetric cuts on the real axis and prescribed asymptotic behavior at $x=0$ and $x \rightarrow \infty$, Eqs. (2.35) and (2.28), respectively [35, 32. Equation (2.33) implies that the energy has the BMN scaling in the thermodynamic limit, $\varepsilon \sim 1 / L$. This relation is in an apparent contradiction with the well-known fact that for $N \gg L$ the anomalous dimension should scale logarithmically $\varepsilon \sim \ln N$ with the prefactor being $L$ independent. To understand the reason for this discrepancy, one notices that the relation (2.33) is valid provided that $S^{\prime}(x)$ is analytical in the vicinity of $x=0$, or equivalently, there is no accumulation of the Bethe roots around the origin. For $N, L \rightarrow \infty$ with $N / L=$ fixed this is indeed the case but, as we will argue in the next section, the situation drastically changes for $N \gg L$.

As a hint, let us consider the eigenstates with $L=$ fixed, $N \rightarrow \infty$ and the charges satisfying (2.31). In this case, the transfer matrix $\tau_{0}(x)$, Eq. (2.16), is an even polynomial of degree $L$ which scales for $x \rightarrow 0$ as

$$
\tau_{0}(x) \sim \frac{\widehat{q}_{L}^{(0)}}{x^{L}}
$$

provided that $\widehat{q}_{L}^{(0)} \neq 0$. If $\widehat{q}_{L}^{(0)}$ vanishes, the asymptotics of $\tau_{0}(x)$ is governed by the first nonvanishing charge $\widehat{q}_{2 k}^{(0)} \neq 0$ with $2 k<L$. Substituting (2.38) into (2.23) one finds the leading asymptotic behavior of the momentum for $x \rightarrow 0$ on the upper sheet of (2.19) as

$$
d p \sim i L \frac{d x}{x}
$$

yielding $p(x) \sim i L \ln x$. According to (2.10), $\beta \sim s L / N \rightarrow 0$ for $N \rightarrow \infty$ and one finds from (2.17) the asymptotics of $S_{0}^{\prime}(x)$ above $x=0$ on the physical sheet of (2.19) as

$$
S_{0}^{\prime}(x) \sim i L \ln x
$$

Comparing this relation with (2.35), one concludes that $S_{0}^{\prime}(x)$ is no longer analytical at the origin due to accumulation of rescaled Bethe roots at $x=0$. Finally, one substitutes (2.40) into (2.13) and obtains the energy

$$
\varepsilon \sim 2 L \ln (L / \beta) \sim 2 L \ln N
$$


Notice that the coefficient in front of $\ln N$ in the right-hand side of (2.41) is determined by the leading asymptotic behavior of the transfer matrix (2.38) for $x \rightarrow 0$. For $\widehat{q}_{L}^{(0)} \neq 0$ this coefficient takes the maximal possible value. For $\widehat{q}_{L}^{(0)}=\ldots=\widehat{q}_{2 m+2}^{(0)}=0$, the transfer matrix (2.16) scales as $\tau_{0}(x) \sim \widehat{q}_{2 m}^{(0)} / x^{2 m}$ (recall that the charges with odd indices vanish, Eq. (2.31)) leading to

$$
\varepsilon \sim 4 m \ln N, \quad \widehat{q}_{2 m+2}^{(0)}=\ldots=\widehat{q}_{L}^{(0)}=0 .
$$

The values of the remaining charges $\widehat{q}_{4}^{(0)}, \ldots, \widehat{q}_{2 m}^{(0)}$ will be determined below (see Eq. (2.48)) Throughout this paper we are interested in the eigenstates with the minimal energy for given $N$. Obviously, they correspond to $m=1$, that is,

$$
\varepsilon^{(m=1)} \sim 4 \ln N, \quad \widehat{q}_{4}^{(0)}=\ldots=\widehat{q}_{L}^{(0)}=0 .
$$

Here the superscript '(0)' refers to the leading order approximation of the semiclassical expansion and $\widehat{q}_{2 k}^{(0)}=0$ does not necessarily imply that $\widehat{q}_{2 k}=0$ but rather $\widehat{q}_{2 k}=\mathcal{O}(\eta)$, or equivalently $q_{2 k} \ll\left(-q_{2}\right)^{k}$.

The transfer matrix $\tau_{0}(x)$, Eq. (2.16), corresponding to the minimal energy state (2.43) is given by

$$
\tau_{0}^{(m=1)}(x)=2-\frac{1}{x^{2}}
$$

and the spectral curve (2.19) looks like

$$
\Gamma_{L}^{(m=1)}: \quad y^{2}=\left(x^{2}-\frac{1}{4}\right) \frac{4}{x^{4}} .
$$

It is easy to see that $\Gamma_{L}^{(m=1)}$ coincides with the complex curve for the spin chain of length 2 , that is $\Gamma_{L=2}$, Eq. (2.19). Indeed, choosing $\widehat{q}_{2 m+1}^{(0)}=\ldots=\widehat{q}_{L}^{(0)}=0$ in (2.19) one effectively descends from an infinitely long spin chain (for $L \rightarrow \infty$ ) to the one with finite length $2 m$. Comparing (2.45) with (2.21) we conclude that for $m=1$ all but two branching points condense at the origin, $b=x_{2 j}=0$, and the two remaining (resolved) branching points are located at $\pm 1 / 2$. For $m \geq 2$, one can show [36] that the complex curve corresponding to (2.42) is given by

$$
\Gamma_{L}^{(m \geq 2)}: \quad y^{2}=\left(x^{2}-\frac{1}{4 m^{2}}\right) \frac{4}{x^{4}} \prod_{j=1}^{m-1}\left(1-\frac{x_{2 j}^{2}}{x^{2}}\right)^{2},
$$

with $1 / x_{2 j}=2 m \cos \left(\frac{\pi j}{2 m}\right)$. Comparison with (2.37) yields $a=1 /(2 m)$ and $b=0$, that is, the two cuts $[-a,-b]$ and $[b, a]$ collide at the origin. Notice that $\Gamma_{L}^{(m \geq 2)}$ has exactly $2 m$ double points satisfying $x_{2 j}^{2}>1 /(2 m)^{2}$ while the remaining double points in (2.37) belonging to the interval $[-b, b]$ condensed at $x=0$ as $b \rightarrow 0$.

The complex curves (2.45) and (2.46) have genus zero and, as a consequence, the momentum $p^{\prime}(x)$ is expressed in terms of elementary functions. Indeed, one substitutes (2.45) and (2.46) into (2.23) and obtains the following expression for the momentum for arbitrary $m$

$$
d p=-\frac{d x}{x \sqrt{x^{2}-\frac{1}{(2 m)^{2}}}}, \quad p(x)=\int_{\infty}^{x} d p=2 m \arcsin \left(\frac{1}{2 m x}\right) .
$$



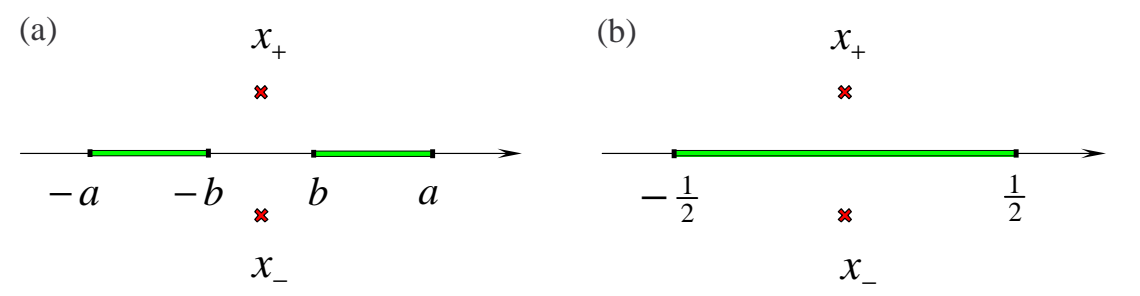

(c)

Figure 2: Symmetric two-cut configuration (a) resulting in the BMN scaling of the anomalous dimension in gauge theory for $\xi<1$. For $\xi \gg 1$ the two cuts collide at the origin (b) yielding the logarithmic scaling. The same configuration in string sigma model (c) for $\xi_{\text {str }} \gg 1$ - the minimal value for the inner end of the cut is given by the BMN coupling $\sqrt{\lambda^{\prime}}$ which prevents the cuts to collide.

Together with (2.17) and (2.16) this leads to the following expressions for the integrals of motion

$$
\widehat{q}_{2 n}^{(0)}=\frac{2(-1)^{n} m^{1-2 n} \Gamma(m+n)}{\Gamma(2 n+1) \Gamma(m-n+1)} .
$$

We conclude from (2.47) that for $m=1$, for the eigenstate with the minimal energy, in the limit $N \rightarrow \infty$ and $L=$ fixed, the momentum $p^{\prime}(x)$ is an analytical function on the complex plane with the square root cut $\left[-\frac{1}{2}, \frac{1}{2}\right]$ and a simple pole at the origin. This should be compared with the analytical properties of the momentum in the region $N / L=$ fixed in which case $p^{\prime}(x)$ has a double pole at $x=0$, Eq. (2.36).

We demonstrated in this section that the analytical properties of the momentum $p(x)$ and the action function $S^{\prime}(x)$ are quite different in the two limits mentioned above. For $0<\beta<1$, the Bethe roots condense on two symmetric intervals $[-a,-b]$ and $[b, a]$ with the end-points $a$ and $b$ depending on $\beta$. For $\beta \rightarrow 0$, one has $b \rightarrow 0$ and $a \rightarrow \frac{1}{2}$ so that the two cuts collide and form a single cut $\left[-\frac{1}{2}, \frac{1}{2}\right]$ (see Fig. 2 (a) and (b)). This leads to different scaling behavior of the energy in these two limits, Eqs. (2.33) and (2.43), respectively. The question remains what happens in the intermediate region of the parameter $\beta=L s /(N+L s)$, Eq. (2.10), and how important the corrections $\sim \eta$ to the energy (2.33) are. We shall demonstrate in the next section that the semiclassical expansion of the energy (2.33) becomes divergent for $\beta \rightarrow 0$ due to the collision of cuts and work out an asymptotic expression for the energy valid for small $\beta$.

\section{Symmetric two-cut solution in thermodynamic limit}

In this section, we construct the minimal energy trajectory in the thermodynamic limit $L \gg 1$ for arbitrary values of the spin $N$. To begin with, we work out the semiclassical expansion of solutions $Q(u)$ to the Baxter equation (2.3) for $N / L=$ fixed and, then, extend the analysis to the region $N \gg L$.

\subsection{Semiclassical expansion at leading order}

To leading order of the semiclassical expansion (2.15) and (2.12), the function $S_{0}^{\prime}(x)$ is determined by the momentum $p(x)$, Eq. (2.17). The latter is fixed by the condition that $d p(x)$ should be a meromorphic differential on the complex curve (2.37) with prescribed asymptotic behavior at 
infinity, Eq. (2.24), and at the origin, Eq. (2.36), on the upper sheet of $\Gamma_{L}$

$$
d p \stackrel{x \rightarrow \infty}{\sim}-\frac{d x}{x^{2}}, \quad d p \stackrel{x \rightarrow 0}{\sim} d x\left[-\frac{\beta}{x^{2}}+\mathcal{O}\left(x^{0}\right)\right]
$$

with $\beta=L s /(N+L s)$, Eq. (2.10). Being combined together, these conditions lead to the following expression [24]

$$
d p=\frac{-1+\frac{\beta a b}{x^{2}}}{\sqrt{\left(x^{2}-a^{2}\right)\left(x^{2}-b^{2}\right)}} d x .
$$

It defines $d p$ as a meromorphic differential on the elliptic curve $\tilde{y}^{2}=\left(x^{2}-a^{2}\right)\left(x^{2}-b^{2}\right)$, Eq. (2.37), which has a pair of double poles above $x=0$ on both sheets of the corresponding Riemann surface. The values of the parameters $a$ and $b$ are fixed by the normalization conditions (2.25)

$$
\int_{b}^{a} d p=0, \quad \int_{a}^{\infty} d p=-\pi m
$$

Here the integer $m$ defines the position of the interval $[b, a]$ inside (2.20). We remind that in the limit we are interested in, the complex curve $\Gamma_{L}$ gets reduced to the genus one curve (2.37). Namely, all cuts in (2.20) except two, $[-a,-b]$ and $[b, a]$, shrink into points. The integer $m-1$ counts how many collapsed cuts are situated to the right from the interval $[b, a]$ on the real axis. The energy depends on $m$ and, as we will see in a moment, it takes the minimal value for $m=1$, that is, when all shrunken cuts are located inside the interval $[-b, b]$.

Solving (3.3) one finds the positions of the cuts as a function of the parameter $\beta$, Eq. (2.10)

$$
a=\frac{1}{2 m \mathbb{E}(\tau)}, \quad b=\frac{\beta}{2 m} \frac{1}{\mathbb{K}(\tau)}, \quad \beta=\sqrt{1-\tau} \frac{\mathbb{K}(\tau)}{\mathbb{E}(\tau)},
$$

where $\mathbb{K}(\tau)$ and $\mathbb{E}(\tau)$ are elliptic integrals of the first and second kind, respectively, and the modular parameter is defined as (for $b \leq a$ )

$$
\tau=1-\frac{b^{2}}{a^{2}}
$$

From (3.2) and (2.17) one obtains the leading term in the semiclassical expansion as

$$
S_{0}^{\prime \prime}(x)=p^{\prime}(x)+\frac{\beta}{x^{2}}=\frac{-1+\frac{\beta a b}{x^{2}}}{\sqrt{\left(x^{2}-a^{2}\right)\left(x^{2}-b^{2}\right)}}+\frac{\beta}{x^{2}},
$$

with the parameters $a$ and $b$ given by (3.4). One verifies that $S_{0}^{\prime \prime}(x)$ has a regular expansion around $x=0$ which is in agreement with our expectations that the Bethe roots condense on the intervals $[-a,-b] \cup[b, a]$ and there is no accumulation of roots at the origin. The energy (2.33) is determined by the leading term in the expansion of this expression [18]

$$
\varepsilon=-\frac{2 \beta}{L}\left[\frac{1}{a b}-\frac{\beta}{2}\left(\frac{1}{a^{2}}+\frac{1}{b^{2}}\right)\right]=\frac{(2 m)^{2}}{L} \mathbb{K}(\tau)[(2-\tau) \mathbb{K}(\tau)-2 \mathbb{E}(\tau)] .
$$

The dependence of $\varepsilon$ on the total spin $N$ and conformal spin $s$ enters into this expression through the parametric dependence of the modular parameter $\tau$, Eq. (3.4), on the scaling parameter $\beta=L s /(N+L s)$, Eq. (2.10). 
We observe that the minimal energy corresponds to $m=1$, that is, when all double points $x_{2 j}$, Eq. (2.37), are located inside the interval $[-b, b]$. It is straightforward to find their position on the real axis with the help of (2.32) and (3.2). Since the total number of double points is infinite for $L \rightarrow \infty$ and they occupy a compact interval, $x_{2 j}$ should be a smooth function of $j$. Differentiating the second relation in (2.32) with respect to $j$, one finds

$$
\frac{d x_{2 j}}{d j}=\frac{\pi}{p^{\prime}\left(x_{2 j}\right)}=-x_{2 j}^{2} \frac{\pi}{\beta}\left[1+\mathcal{O}\left(x_{2 j}^{2}\right)\right],
$$

where we substituted the momentum by its value at the origin (3.1). It follows from (3.8) that for large $j$ the double points $1 / x_{2 j}$ are equidistantly distributed on the real axis (see Fig. 3)

$$
\frac{1}{x_{2 j}}=\frac{\pi}{\beta} j+\mathcal{O}(1 / j)
$$

We remind that the double points $x_{2 j}$ verify the relation $\tau_{0}\left(x_{2 j}\right)= \pm 2$. It is instructive to compare (3.9) with a similar relation $1 / x_{2 j}=\pi j$ for the momentum (2.32) corresponding to the state with $N=0$, or equivalently $\beta=1$. Equation (3.9) suggests that close to the origin and away from the cuts, $x^{2} \ll b^{2}$, the transfer matrix for the two-cut solution looks like $\tau_{0}(x)=$ $2 \cos p(x) \sim 2 \cos (\beta / x)$. Matching this relation into the general expression for $\tau_{0}(x)$, Eq. (2.14), we find that the integrals of motion scale as

$$
\widehat{q}_{2 n} \sim 2 \frac{(-1)^{n}}{(2 n) !} \beta^{2 n}, \quad(n \gg 1)
$$

where $\widehat{q}_{2 n}=q_{2 n} /(N+L s)^{2 n}$. For $\beta=1$, or equivalently $N=0$, this relation is exact for all $\widehat{q}_{2 k}$. For $\beta \rightarrow 0$, or equivalently $N \rightarrow \infty$, it cannot be exact since, by definition, $\widehat{q}_{2}=-1+\mathcal{O}(\eta)$. It instead indicates that higher charges take anomalously small values $\widehat{q}_{2 k}=\mathcal{O}\left(\eta^{2 k}\right)$ with $\eta \sim 1 / N$. This is in agreement with our expectations that for $\beta=0$ the transfer matrix reduces to (2.44).

Let us examine the expression for the energy (3.7) in two limiting cases $\beta \rightarrow 1$ and $\beta \rightarrow 0$, or equivalently $N \ll L$ and $N \gg L$, respectively, Eq. (2.11). According to (3.4), the corresponding values of the modular parameter are $\tau \rightarrow 0$ and $\tau \rightarrow 1$. For $\tau \rightarrow 0$, one finds from (3.4)

$$
a=\frac{1}{\pi m}\left(1+\frac{\tau}{4}\right)+\ldots, \quad b=\frac{1}{\pi m}\left(1-\frac{\tau}{4}\right)+\ldots, \quad \beta=1-\frac{\tau^{2}}{16}+\ldots,
$$

where the ellipses denote subleading terms. Since $a-b \sim \tau \rightarrow 0$, the two cuts shrink into points as $\beta \rightarrow 1$. One expands (3.7) in powers of $\tau$ and obtains the energy as

$$
\varepsilon \stackrel{L \gg N}{=} \frac{m^{2}}{8 L} \pi^{2} \tau^{2}+\ldots=\frac{N}{L^{2}} \frac{2 \pi^{2} m^{2}}{s}+\ldots
$$

For $\tau \rightarrow 1$, one has from (3.4)

$$
a=\frac{1}{2 m}+\ldots, \quad b=\frac{\sqrt{1-\tau}}{2 m}+\ldots, \quad \beta=\sqrt{1-\tau} \ln \frac{1}{\sqrt{1-\tau}}+\ldots
$$

According to (2.10), the limit $\beta \rightarrow 0$ corresponds to $\beta=L s / N$ with $N \gg L$. Then, making use of (3.7) one finds for the energy

$$
\varepsilon \stackrel{L \ll N}{=} \frac{4 m^{2}}{L} \ln ^{2} \frac{\sqrt{1-\tau}}{4}+\ldots=\frac{4 m^{2}}{L} \ln ^{2} \frac{N}{L}+\ldots
$$

This relation matches the expression for the energy of long spinning string folded $m$ times [1] and agrees with the thermodynamic Bethe ansatz analysis [18. 


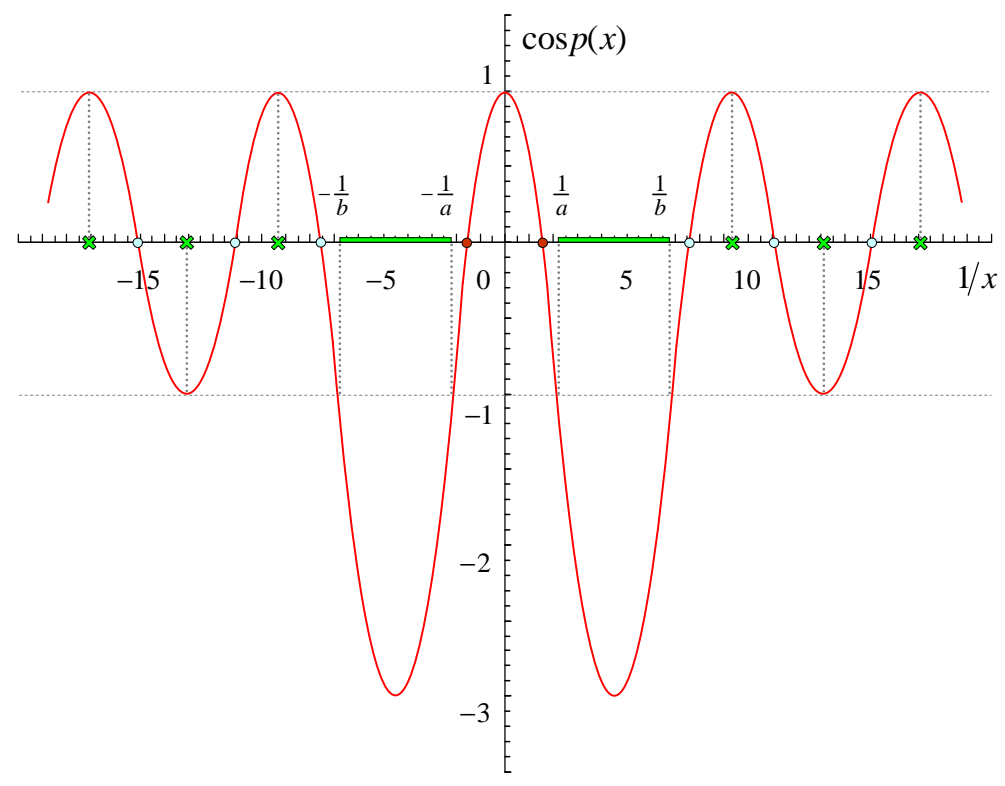

Figure 3: The transfer matrix $\cos p(x)=\tau_{0}(x) / 2$ as a function of $1 / x$ for the symmetric two-cut solution for $\beta=3 / 4$ and $m=1$. The cuts $[-a,-b] \cup[b, a]$ with $a=0.45$ and $b=0.15$ correspond to $\cos ^{2} p(x)>1$. The double points $x_{2 j}$ are denoted by crosses, $\cos p\left(x_{2 j}\right)= \pm 1$ and $1 / x_{2 j}^{2}>1 / b^{2}$. The "large" and "small" roots of the transfer matrix, $\cos p\left(\delta_{n}\right)=0$, are shown by full and light blobs, respectively.

\subsection{Breakdown of semiclassical expansion}

For $m=1$ the expressions (3.7), (3.12) and (3.14) describe the dependence of the energy on the total spin $N+L s$ along the minimal energy trajectory at leading order of the semiclassical expansion for $L \gg 1$ and arbitrary values of $N$ including the regions $N \ll L$ and $N \gg L$. From (2.33) one expects that the contribution of the subleading terms to the energy is suppressed by powers of $\eta=1 /(L+N s)$. Let us compare these predictions with the exact expressions for the minimal energy obtained from numerical solution to the Baxter equation, Eqs. (2.3) and (2.8). For $L=10$, the comparison is shown in Fig. 4 on the right panel. We observe that the curve determined by (3.7) agrees with the exact numbers for $N<L$ and deviates significantly from them for $N>L$. Most importantly, for $N \gg L$ the exact energy scales as $\sim 4 \ln N$ while the semiclassical formula (3.14) yields a different asymptotic behavior.

To understand the reason for this discrepancy, let us revisit the calculation of the energy in the previous section and pay special attention to the position of the cuts. By construction, the cuts run along two symmetric intervals $[-a,-b] \cup[b, a]$. According to (3.13), for $\tau \rightarrow 1$ the parameter $a$ approaches the value $a=1 /(2 m)$ while the parameter $b$ vanishes indicating that the two cuts collide at the origin. Then, one examines the expression for the momentum (3.2) and finds that for $b=0$ the differential $d p$ reduces to (2.47). We argued in Sect. 2.3 that Eq. (2.47) leads to a new, logarithmic scaling of the energy (2.43). This suggests that the problem with recovering this regime within the semiclassical approach does not lie in the construction of the momentum $p(x)$ but rather in the expression for the energy (2.33).

The exact formula for the energy (2.8) involves $S^{\prime}\left(x_{ \pm}\right)$evaluated at $x_{ \pm}= \pm i \beta / L$. The relation (2.33) was derived under the assumption that $S^{\prime}(x)$ is analytical at the vicinity of these points. 
For the two-cut solution constructed in the previous section this assumption is justified provided that the inner boundary of the cut $b$ stays finite in the limit $L \rightarrow \infty$. For $x_{ \pm} \rightarrow 0$ with $b=$ fixed, the semiclassical analysis is applicable and one arrives at the asymptotic behavior (3.12) and (3.14) depending on the ratio $N / L$. The situation is more complex when $b$ vanishes in the scaling limit. For $b \rightarrow 0$ with $x_{ \pm}=$fixed, the two cuts collide and the very assumption that $S^{\prime}(x)$ is analytical at the origin does not hold true anymore. In this case, one expects that the energy will develop a new asymptotic behavior and the crossover will occur for $b \sim\left|x_{ \pm}\right|$(see Fig. 2 (a) and (b)).

Defining a new parameter $\xi=\left|x_{ \pm}\right| / b=\beta /(b L)$ one anticipates that the semiclassical approach is applicable for $\xi<1$. Indeed, for $N \ll L$ one finds from (3.11) that $\xi \sim 1 / L$ and the condition $\xi<1$ is satisfied. For $N \gg L$ one gets from (3.11)

$$
\xi \sim \frac{1}{L} \ln \frac{N}{L} .
$$

One recognizes that similar parameter $\xi_{\text {str }}$ naturally arises on the string side and controls there the transition between two different regimes in (1.4). For $\xi \gg 1$ one has to take into account that the two cuts collide at the origin and, as a consequence, the analytical properties of $S^{\prime}(x)$ are different. The simplest way to see this is to examine the Taylor expansion of the leading term $S_{0}^{\prime \prime}(x)$, Eq. (3.6), around the origin and retain the terms most singular for $b \rightarrow 0$

$$
S_{0}^{\prime \prime}(x) \sim \frac{\beta}{x^{2}}\left[1-\frac{1}{\sqrt{1-x^{2} / b^{2}}}\right]=-\frac{\beta}{2 b^{2}}\left[1+\frac{3}{4} \frac{x^{2}}{b^{2}}+\frac{5}{8} \frac{x^{4}}{b^{4}}+\ldots\right] .
$$

As follows from (2.14) and (2.33), higher order terms of this expansion contribute to subleading corrections to the energy $\varepsilon=i \int_{x_{-}}^{x_{+}} d x S^{\prime \prime}(x)$. It is easy to see that the series for $i \int_{x_{-}}^{x_{+}} d x S_{0}^{\prime \prime}(x)$ runs in powers of $\xi=\left|x_{ \pm} / b\right|$ and it is only convergent for $\xi<1$.

Based on our analysis we expect that the semiclassical expansion of the energy (2.33) will be divergent for $\xi \gg 1$. Let us calculate the first subleading correction to the energy (2.33) defined by $S_{1}^{\prime \prime}(0)$. The function $S_{1}^{\prime}(x)$ satisfies the relation (2.18) which involves yet unknown function $\tau_{1}(x)$. To determine $S_{1}^{\prime}(x)$ one requires that it should have the same analytical properties as the leading term of the semiclassical expansion $S_{0}^{\prime}(x)$, i.e., to be an analytical function in the complex plane with the cuts $[-a,-b] \cup[b, a]$. Discontinuity of $S_{1}^{\prime}(x)$ across the cut defines the correction to the distribution density of the Bethe roots [26]. Since $\tau_{0}(x)$ and $\tau_{1}(x)$ are entire functions while $p^{\prime}(x)$ and $\sin p(x)$ change sign across the cut, $\sin p(x+i 0)=-\sin p(x-i 0)$, the function $S_{1}^{\prime}(x)$ satisfies the relation

$$
S_{1}^{\prime}(x+i 0)+S_{1}^{\prime}(x-i 0)=-p^{\prime}(x+i 0) \cot p(x+i 0),
$$

with $x \in[-a,-b] \cup[b, a]$. In addition, its asymptotic behavior at the origin and infinity is fixed by Eqs. (2.28) and (2.35),

$$
S_{1}^{\prime}(0)=0, \quad S_{1}^{\prime}(x) \stackrel{x \rightarrow \infty}{\sim} \frac{1}{x^{3}} .
$$

The solution to the resulting Riemann-Hilbert problem looks like (c.f. [37])

$$
S_{1}^{\prime}(x)=\frac{x}{\sqrt{\left(x^{2}-a^{2}\right)\left(x^{2}-b^{2}\right)}} \int_{b}^{a} \frac{d z}{\pi} \frac{\sqrt{\left(a^{2}-z^{2}\right)\left(z^{2}-b^{2}\right)}}{x^{2}-z^{2}} p^{\prime}(z+i 0) \cot p(z+i 0),
$$


where ' $+i 0$ ' indicates that the momentum is evaluated on the upper sheet of the Riemann surface. Replacing $p^{\prime}(z+i 0)$ by its expression (3.2), we obtain the following representation for the first subleading correction to (2.33)

$$
\varepsilon_{1}=-\frac{2 \beta}{L} S_{1}^{\prime \prime}(0)=\frac{2 \beta}{\pi a b L} \int_{b}^{a} \frac{d z}{z^{2}}\left[1-\beta \frac{a b}{z^{2}}\right] \operatorname{coth}(i p(z+i 0)) .
$$

For our purposes we would like to determine the asymptotic behavior of $\varepsilon_{1}$ for $N \gg L$, or equivalently $a \rightarrow 1 /(2 m), b \rightarrow 0$ and $\beta \rightarrow 0$, Eq. (3.13). Changing the integration variable to $z \rightarrow b z$, one finds in the limit $b \rightarrow 0$

$$
\varepsilon_{1}=\frac{2 \beta}{\pi a b^{2} L} \int_{1}^{\infty} \frac{d z}{z^{2}}\left[1-\frac{\beta a}{b} \frac{1}{z^{2}}\right] \operatorname{coth}(i p(b z+i 0))=-\frac{2}{3 \pi} \frac{\beta^{2}}{L b^{3}}+\ldots
$$

Here in the second relation we took into account that for $\beta \rightarrow 0$ the cuts collide at the origin and the momentum scales as $p(z+i 0) \sim 2 i m \ln z$ for $z \rightarrow 0$, Eq. (2.47). Comparing (3.21) with a similar relation for the leading term $\varepsilon_{0} \sim \beta^{2} /\left(L b^{2}\right)$, Eq. (3.7), one finds that

$$
\frac{\eta \varepsilon_{1}}{\varepsilon_{0}}=\mathcal{O}(\eta / b)=\mathcal{O}(\xi)
$$

where $\eta / b=1 /(b N)=(2 m / s) \ln (N / L) / L$ in the limit $N \gg L$, Eqs. (2.10) and (3.13).

We conclude that for $N \gg L$ the semiclassical expansion of the energy, $\varepsilon=\varepsilon_{0}+\eta \varepsilon_{1}+\mathcal{O}\left(\eta^{2}\right)$, Eq. (2.33) runs in powers of $\xi$ and it is only convergent for $\xi<1$. Together with (2.2) this leads to the expression for the one-loop anomalous dimension, Eqs. (1.7) and (1.8), with $c_{1,1}=$ $-2 m /(3 \pi s)$.

\subsection{Beyond semiclassical expansion}

We demonstrated in the previous section that the semiclassical expansion breaks down for $\xi \gg 1$. The reason for this is that the cuts collide in this limit and the semiclassical expression for $S(x)$ ceases to be analytical at $x=0$. Substituting $\beta=0$ into (3.6), one finds (for $m=1$ )

$$
S_{0}^{\prime \prime}(x)=p^{\prime}(x)=-\frac{1}{x \sqrt{x^{2}-\frac{1}{4}}}
$$

and, therefore, $S_{0}^{\prime}(x) \sim \mp 2 i \ln x$ for $x \rightarrow 0$ on the upper and lower sheets, respectively. It is easy to see that the corresponding transfer matrix $\tau_{0}(x)=2 \cos p(x)$ is given by (2.44). We remind that $\tau_{0}(x)$ plays the role of the potential in the Baxter equation (2.3). The fact that $\tau_{0}(x)$ is singular at $x=0$ implies that the semiclassical expansion (2.15) breaks down at the origin. In this section, we present an approach which allows one to construct asymptotic solution to the Baxter equation (2.3) and evaluate the energy for $N>L$. It is complementary to the semiclassical approach and takes a full advantage of the above mentioned singularity of the transfer matrix $\tau_{0}(x)$ at $x=0$. A detailed account on this approach can be found in Ref. [15].

To begin with, we rewrite the Baxter equation (2.3) as

$$
(u+i s)^{L} \phi(u)+\frac{(u-i s)^{L}}{\phi(u-i)}=t_{L}(u)
$$


where the notation was introduced for

$$
\phi(u)=\frac{Q(u+i)}{Q(u)}
$$

To evaluate the energy (2.8) one has to construct solutions to (3.24) for $u \sim \pm i s$. Notice that the dressing factors $(u \pm i s)^{L}$ in (3.24) vanish for $u \rightarrow \mp i s$ indicating that one of the terms in the left-hand side of (3.24) becomes anomalously small and can be neglected. It turns out that in the thermodynamic limit, $L \gg 1$, the same approximation can be performed not only in the vicinity of $u= \pm i s$ but in the whole region $u=\mathcal{O}\left((N+L s)^{0}\right)$. To see this we note that $t_{L}(u)$ is given by (2.4) with the charges that scale as $q_{k} \sim(N+L s)^{k}$. This suggests that $\left|t_{L}(u)\right| \gg 1$ for $u=\mathcal{O}\left((N+L s)^{0}\right)$. Indeed, in the semiclassical approach, for $u=x / \eta$, one finds from (2.14), (2.17) and (3.23) that the transfer matrix takes the form

$$
t_{L}(x / \eta)=2(x / \eta)^{L} \cos p(x)+\mathcal{O}(\eta) .
$$

For $u=\mathcal{O}\left(L^{0}\right)$, or equivalently $x \sim \eta$, the momentum can be replaced by its asymptotic behavior at the origin: $p(x) \sim \beta / x$ for $\xi \ll 1$ and $p(x) \sim 2 i \ln x$ for $\xi \gg 1$ leading to $\left|t_{L}(x / \eta)\right| \gg 1$.

Let us return to Eq. (3.24) and take into account that $t_{L}(u)$ takes large values for $u=$ $\mathcal{O}\left((N+L s)^{0}\right)$ both for $\xi<1$ and $\xi \gg 1$. Requiring the left-hand side of (3.24) to be as large as $t_{L}(u)$ one finds that either $\phi(u) \gg 1$, or $\phi(u-i) \ll 1$. In both cases, one of the terms in the left-hand side of (3.24) can be safely neglected and one arrives at two different equations

$$
\begin{aligned}
& (u+i s)^{L} Q_{+}(u+i)=t_{L}(u) Q_{+}(u), \\
& (u-i s)^{L} Q_{-}(u-i)=t_{L}(u) Q_{-}(u) .
\end{aligned}
$$

Having solved this system, one can construct an asymptotic solution to the Baxter equation (2.3) as a linear combination of $Q_{+}(u)$ and $Q_{-}(u)$

$$
Q^{(\mathrm{as})}(u)=c_{+} Q_{+}(u)+c_{-} Q_{-}(u) .
$$

We would like to stress that $Q^{(\text {as) }}(u)$ does not satisfy the Baxter equation (2.6), but asymptotically approaches its solution $Q(u)$ in the region $u=\mathcal{O}\left((N+L s)^{0}\right)$. Equations (3.27) were obtained under the assumption that $(u+i s)^{L} Q_{+}(u+i) \gg(u-i s)^{L} Q_{+}(u-i)$ and $(u+i s)^{L} Q_{-}(u+i) \ll$ $(u-i s)^{L} Q_{-}(u-i)$, respectively. Together with (3.27) it can be expressed as the following relation for the transfer matrix

$$
t_{L}\left(u+\frac{i}{2}\right) t_{L}\left(u-\frac{i}{2}\right) \gg\left[u^{2}+\left(\frac{1}{2}-s\right)^{2}\right]^{L} .
$$

For $u=\mathcal{O}\left((N+L s)^{0}\right)$ it is equivalent to $\left|t_{L}(u)\right| \gg 1$.

To solve (3.27) one introduces into consideration the roots of the transfer matrix (2.4)

$$
t_{L}(u)=2 \prod_{n=1}^{L}\left(u-\delta_{n}\right)
$$

It is known that for polynomial solutions to the Baxter equation, Eq. (2.6), the roots take real values only, $\operatorname{Im} \delta_{k}=0$ [26]. Matching (3.30) into (2.4) one finds that they satisfy the sum rules

$$
\sum_{n=1}^{L} \delta_{n}=0, \quad \sum_{n=1}^{L} \delta_{n}^{2}=-\frac{1}{2} q_{2}, \quad \ldots, \quad \prod_{n=1}^{L} \delta_{n}=\frac{(-1)^{L}}{2} q_{L} .
$$


For even solutions to the Baxter equation, $Q(u)=Q(-u)$, the roots appear in pairs $\delta_{n}=$ $-\delta_{L-n+1}$. Making use of (3.30), it is straightforward to verify that the solutions to (3.27) are given by

$$
Q_{+}(u)=2^{-i u} \prod_{n=1}^{L} \frac{\Gamma\left(-i u+i \delta_{n}\right)}{\Gamma(-i u+s)}, \quad Q_{-}(u)=2^{i u} \prod_{n=1}^{L} \frac{\Gamma\left(i u-i \delta_{n}\right)}{\Gamma(i u+s)}
$$

To fix the constants $c_{ \pm}$in (3.28) one examines the relation for the quasimomentum in Eq. (2.8) and substitutes $Q(u)$ by its asymptotic expression (3.28). Taking into account that (for $\varrho \rightarrow 0$ )

$$
Q_{+}(-i s+\varrho) \sim \varrho^{L}, \quad Q_{-}(i s-\varrho) \sim \varrho^{L}
$$

one finds from (2.8) and (3.28)

$$
\mathrm{e}^{i \theta}=\frac{Q^{(\mathrm{as})}(i s)}{Q^{(\mathrm{as})}(-i s)}=\frac{c_{+}}{c_{-}} \frac{Q_{+}(i s)}{Q_{-}(-i s)} .
$$

Therefore, for cyclically symmetric states $\mathrm{e}^{i \theta}=1$ the asymptotic solution to the Baxter equation is given up to an overall normalization factor by

$$
Q^{(\mathrm{as})}(u)=Q_{+}(u) Q_{-}(-i s)+Q_{-}(u) Q_{+}(i s) .
$$

We remind that this relation is only valid in the region $u=\mathcal{O}\left((N+L s)^{0}\right)$. In a similar manner, one uses (3.33) to evaluate the energy (2.8) as

$$
\varepsilon^{(\mathrm{as})}=i\left(\ln Q^{(\mathrm{as})}(i s)\right)^{\prime}-i\left(\ln Q^{(\mathrm{as})}(-i s)\right)^{\prime}=i\left(\ln Q_{+}(i s)\right)^{\prime}-i\left(\ln Q_{-}(-i s)\right)^{\prime} .
$$

Replacing $Q_{ \pm}(u)$ by their expressions (3.32) one finds the following remarkable expression for the energy in terms of the roots of the transfer matrix (3.30) [26, 13, 14, 15]

$$
\varepsilon^{(\mathrm{as})}=2 \ln 2+\sum_{n=1}^{L}\left[\psi\left(s+i \delta_{n}\right)+\psi\left(s-i \delta_{n}\right)-2 \psi(2 s)\right],
$$

where $\psi(x)=d \ln \Gamma(x) / d x$. The interpretation of (3.37) in terms of classical $S L(2)$ spin magnet and properties of the Wilson operators (2.1) in gauge theory can be found in Ref. [8].

Since the roots $\delta_{n}$ are functions of the conserved charges, Eq. (3.31), the relation (3.37) establishes the dependence of the energy on $q_{2}, \ldots, q_{L}$. To check (3.37), we compared the asymptotic "dispersion curve" $\varepsilon^{(\mathrm{as})}\left(q_{2}, \ldots, q_{L}\right)$ with the exact one $\varepsilon^{(\mathrm{ex})}\left(q_{2}, \ldots, q_{L}\right)$ coming from the Bethe Ansatz solution (2.9). We found that for $L=10$ and $s=1 / 2$ the accuracy of (3.37), $\varepsilon^{(\text {as })} / \varepsilon^{(\mathrm{ex})}-1$, increases from $-4.6 \times 10^{-5}$ for $N=2$, to $-2.6 \times 10^{-6}$ for $N=10$ and to $1.9 \times 10^{-10}$ for $N=100$. We conclude that Eq. (3.37) describes the exact eigenspectrum with a high accuracy throughout the whole interval of $N$ including the region $N \sim L$ in which the semiclassical approach is applicable. Indeed, it is straightforward to show that the relation (3.37) coincides with the semiclassical expression (2.33) for $N \sim L$ (see Appendix).

The charges $q_{3}, \ldots, q_{L}$ have to satisfy quantization conditions. In the method of Baxter $Q$-operator they follow from the requirement for $Q(u)$ to be polynomial solutions to the Baxter equation, Eqs. (2.3) and (2.6). The roots of $Q(u)$ scale in the thermodynamic limit as $u=\lambda_{j} \sim L$ and, therefore, they lie outside the applicability range of (3.35). This does not allow us to impose 
the polynomiality condition for $Q^{(\text {as) }}(u)$. We shall require instead that $Q^{(\text {as) }}(u)$, given by (3.35), should be regular on the real $u$-axis inside the region $u=\mathcal{O}\left((N+L s)^{0}\right)$. This property is not warranted since both $Q_{+}(u)$ and $Q_{-}(u)$ develop poles on the real $u$-axis originating from the product of $\Gamma$-functions in the numerator in the right-hand side of Eq. (3.32). The poles are located at zeros of the transfer matrix (3.30),$u=\delta_{j}$ with $j=1, \ldots, L$. Requiring for $Q^{(\text {as) }}(u)$ to have zero residues at $u=\delta_{n}$ with $\delta_{n}=\mathcal{O}\left((N+L s)^{0}\right)$, one gets from (3.35) and (3.32)

$$
2^{-2 i \delta_{n}} \prod_{j=1, j \neq n}^{L} \frac{\Gamma\left(-i \delta_{n}+i \delta_{j}\right)}{\Gamma\left(i \delta_{n}-i \delta_{j}\right)}=\left[\frac{\Gamma\left(s-i \delta_{n}\right)}{\Gamma\left(s+i \delta_{n}\right)}\right]^{L} \prod_{j=1}^{L} \frac{\Gamma\left(s+i \delta_{j}\right)}{\Gamma\left(s-i \delta_{j}\right)}
$$

The total number of roots of the transfer matrix (3.30) equals the length of the spin chain $L$. As we will see in a moment, in the thermodynamic limit $L \gg 1$ and $N \gg L$ all roots can be separated into two different groups depending on their scaling: "small" roots $\delta_{n}=\mathcal{O}\left(N^{0}\right)$ and "large" roots $\delta_{n}=\mathcal{O}(N)$. It is important to realize that the quantization conditions (3.38) should only hold for the small roots while the product over $j$ entering both sides of (3.38) involves all roots.

The total number of small roots depends on the value of the parameter $\beta=L s /(N+L s)$. For $0<\beta \leq 1$, that is, within the applicability range of the semiclassical expansion, the integrals of motion scale as in Eq. (3.10). Together with (3.31) this implies that the roots behave as $\delta_{n}=\mathcal{O}(N)$ and, therefore, there are no small roots. For $\beta \rightarrow 0$ higher charges $q_{L}, q_{L-1}, \ldots$ take anomalously small values (3.10) indicating that the small roots are there. Let us demonstrate that their total number equals $L-2 m$ with positive integer $m$ entering the right-hand side of (3.3). One makes use of (3.26) to deduce that the large roots of the transfer matrix satisfy $\cos p\left(\delta_{j} \eta\right)=0+\mathcal{O}(\eta)$. Recall that the momentum verifies $(\cos p(x))^{2} \geq 1$ on the cuts (for $\left.b^{2} \leq x^{2} \leq a^{2}\right)$ and takes the values $\cos p(x)= \pm 1$ at the double points $x=x_{2 j}$, Eq. (3.9). Since $\cos p\left(x_{2 j}\right)=-\cos p\left(x_{2 j-2}\right)$, the roots of the transfer matrix (3.26) should lie on the real axis in between the branching points, $x_{2 k}<\delta_{j} \eta<x_{2 j-2}$, away from the cuts $[-a,-b] \cup[b, a]$ (see Fig. [3]). Going over to the limit $\beta=L s /(N+L s) \rightarrow 0$, or equivalently $N \gg L$, one takes into account that the edges of the cuts scale as $a=1 /(2 m)$ and $b=\beta /(-2 m \ln \beta) \rightarrow 0$, Eq. (3.13). As a result, all roots of the transfer matrix $\delta_{j} \eta \sim \delta_{j} / N$ "trapped" inside the interval $[-b, b]$ are to be small while the roots belonging to the intervals $(-\infty,-a] \cup[a, \infty)$ are to be large

$$
\left|\delta^{(\text {large })}\right|>\frac{N}{2 m}, \quad \quad\left|\delta^{\text {(small })}\right|<\frac{1}{4 m \xi},
$$

with $\xi=\ln (N / L) / L$. Thus, the total number of small roots, $L-2 m$, equals the number of double points on the interval $[-b, b]$ and, as a consequence, the total number of the large roots is $2 m$.

Let us consider the minimal energy state with $m=1$. For $N \gg L$, the transfer matrix has two large roots $\delta_{1}=-\delta_{L}$ and $L-2$ small roots $\delta_{k}=-\delta_{L-k+1}$ with $k=2, \ldots, L-1$. It follows from (3.31) that the large root is given by

$$
\delta_{1}=\left(-q_{2} / 2\right)^{1 / 2}[1+\mathcal{O}(1 / L)] \sim \frac{N+L s}{\sqrt{2}}
$$

with $q_{2}$ defined in (2.5). The small roots satisfy the quantization conditions (3.38). Separating in (3.38) the contribution of the large roots with $j=1$ and $j=L$, one can rewrite (3.38) in 
terms of the small roots only (for $n=2, \ldots, L-1$ )

$$
\left(-q_{2}\right)^{-2 i \delta_{n}}=(-1)^{L-1}\left[\frac{\Gamma\left(s-i \delta_{n}\right)}{\Gamma\left(s+i \delta_{n}\right)}\right]^{L} \prod_{j=2}^{L-1} \frac{\Gamma\left(1+i \delta_{n}-i \delta_{j}\right)}{\Gamma\left(1-i \delta_{n}+i \delta_{j}\right)}
$$

Here we took into account that the product over $j$ in the right-hand side of (3.38) equals 1 due to pairing of the roots $\delta_{n}=-\delta_{L-n+1}$. Taking the logarithm of both sides in (3.41), one rewrites the quantization conditions as

$$
\delta_{n} \ln \left(-q_{2}\right)+L \arg \Gamma\left(s-i \delta_{n}\right)+\sum_{j=2}^{L-1} \arg \Gamma\left(1+i \delta_{n}-i \delta_{j}\right)=\frac{\pi}{2} k_{n}
$$

where integers $k_{2}>k_{3}>\ldots$ count the branches of the logarithms and satisfy $k_{n}=-k_{L-n+1}$. In addition, they have a parity opposite to that of $L$, that is $k_{n}=$ even/odd for $L=$ odd/even. Notice that in distinction with the Bethe ansatz equations (2.7), the number of relations in (3.42) does not depend on the total spin $N$ but only on the length of the spin chain $L$.

To evaluate the energy (3.37) one has to solve the system (3.42) and determine the set of small roots. The resulting expression for $\varepsilon^{(\text {as })}$, Eq. (3.37), depends on integers $k_{n}$ and as we show below (see Eq. (3.48)) it takes minimal value for the occupation numbers $k_{n}=L+1-2 n$. A systematic analysis of the quantization conditions (3.42) will be given elsewhere. The system of equations (3.42) can be easily solved in two limits $\xi \gg 1$ and $\xi<1$.

For $\xi \gg 1$ (or $\ln (N / L) \gg L)$ one deduces from (3.39) that solutions to (3.42) have to satisfy $\left|\delta_{n}\right| \ll 1$. In this case, one expands the $\Gamma$-functions in (3.42) in powers of $\delta$ 's and one finds after some algebra

$$
\delta_{n}=\frac{\pi k_{n} / 2}{\ln \left(-q_{2}\right)+(L-2) \psi(1)-L \psi(s)}+\ldots
$$

with $n=2, \ldots, L-1$. One verifies a posteriori that this relation is in agreement with (3.39).

For $\xi<1$ (or $1 \ll \ln (N / L)<L$ ), one obtains from (3.39) that $\left|\delta_{n}\right|<1 /(2 \xi)$ and, therefore, not all roots verify the relation $\left|\delta_{n}\right| \ll 1$. Still, the relation (3.43) is valid for the roots with small absolute value $\left|\delta_{n}\right| \ll 1$ (with $n \sim L / 2$ ). For roots $\left|\delta_{n}\right| \sim 1 /(2 \xi)$ (with $n \sim L^{0}$ ) one expands the $\Gamma$-functions in (3.42) in inverse powers of $\delta$ 's and obtains

$$
\delta_{n} \ln \left(-q_{2}\right)-\delta_{n} \ln \delta_{n}^{2}+\ldots=\frac{\pi}{2} k_{n}
$$

with $k_{n}=\mathcal{O}(L)$. From this relation one obtains the relation $\delta_{n} \sim \pi k_{n} /\left(4 \ln \left(N / k_{n}\right)\right)$ which is in agreement with (3.39).

To test the quantization conditions (3.42) we compared solutions to (3.42) for $k_{n}=L+1-2 n$ with the exact, Bethe ansatz expressions for the roots of the transfer matrix corresponding to the minimal energy state with the quantum numbers $s=\frac{1}{2}, L=10$ and $N=100$ and observed a good agreement (see Table 11). In agreement with (3.43) and (3.44), the small roots $\delta_{n}$ vary linearly with $n$ close to the origin and deviate from the linear behavior close to the end points. Moreover, for $\xi \gg 1$ all small roots scale linearly with $n$ in agreement with (3.43) (see Fig. 4).

In a similar manner, we solved quantization conditions (3.42) for $s=\frac{1}{2}, L=10$ and $0 \leq N \leq$ 100 and compared the resulting expression for the energy (3.37) with the exact expression (2.9) as shown in Table 2. We found that for $N \geq L$ the asymptotic expression (3.37) approximates the exact result with an accuracy better than $0.002 \%$ while the semiclassical approach significantly 


\begin{tabular}{|c||c|c|c|c|c||c|}
\hline & $\delta_{1}$ & $\delta_{2}$ & $\delta_{3}$ & $\delta_{4}$ & $\delta_{5}$ & $E$ \\
\hline exact & 73.897289 & 0.5297596 & 0.3443964 & 0.1937491 & 0.06253897 & 7.3790455 \\
\hline asym & 73.900271 & 0.5297573 & 0.3443955 & 0.1937487 & 0.06253886 & 7.3791719 \\
\hline
\end{tabular}

Table 1: Comparison of the exact roots, $\delta_{n}=-\delta_{L-n+1}$, and the energy, $E$, for $s=1 / 2, L=10$ and $N=100$ with the asymptotic expressions obtained from Eqs. (3.41) and (3.37), respectively.

\begin{tabular}{|c||c|c|c|c|c|c|c|c|c|c|}
\hline$N$ & 0 & 10 & 20 & 30 & 40 & 50 & 60 & 70 & 80 & 90 \\
\hline exact & 0 & 2.2766 & 3.5069 & 4.3644 & 5.0266 & 5.5678 & 6.0262 & 6.4243 & 6.7765 & 7.0924 \\
\hline asym & 0.0907 & 2.2851 & 3.5097 & 4.3657 & 5.0274 & 5.5683 & 6.0265 & 6.4246 & 6.7767 & 7.0926 \\
\hline
\end{tabular}

Table 2: Comparison of the exact energy, $\varepsilon$, with the asymptotic expression obtained from Eqs. (3.41) and (3.37) for $s=\frac{1}{2}, L=10$ and different total spin $N$.

overestimates the value of the energy (see Fig. 4). Remember that the minimal energy states carry even Lorentz spin only, Eq. (2.31).

Let us examine the expression for the energy (3.37) in the limit $\xi \gg 1$, or $\ln (N / L) \gg L$. We remind that the semiclassical expansion breaks down in this region. Equation (3.37) involves the sum over the roots of the transfer matrix (3.30). As before, we separate them into two groups. According to (3.39), the large and small roots scale as $\delta_{j} \sim N$ and $\delta_{j} \sim 1 / \xi$, respectively. This allows one to replace $\psi\left(s \pm i \delta_{j}\right)$ in (3.37) by its asymptotic behavior at infinity and at the origin, respectively. In this way we obtain

$$
\varepsilon=2 \ln 2-2 L \psi(2 s)+2 \sum_{\text {large }} \ln \left|\delta_{j}\right|+\sum_{\text {small }}\left[2 \psi(s)-\psi^{\prime \prime}(s) \delta_{j}^{2}\right]+\ldots
$$

where the ellipsis denotes subleading terms. The number of large roots equals $2 m, \delta_{j}=-\delta_{L-j+1}$ (with $j=1, \ldots, m$ ) and, therefore, the leading asymptotic behavior of the energy for $\xi \gg 1$ is [8]

$$
\varepsilon=4 \ln \left(\delta_{1} \delta_{2} \ldots \delta_{m}\right)+\mathcal{O}\left(N^{0}\right)=2 \ln \left|q_{2 m}\right|+\mathcal{O}\left(N^{0}\right),
$$

where we took into account the relations (3.31) and $q_{2 m}=\widehat{q}_{2 m}^{(0)} N^{2 m}$ was defined in (2.48). In this way we obtain the relation

$$
\varepsilon=4 m \ln N+\mathcal{O}\left(N^{0}\right),
$$

which is in an agreement with (2.42). The minimal energy corresponds to $m=1$.

For $m=1$ one has just two large roots $\delta_{1}=-\delta_{L}$ and $L-2$ small roots $\delta_{n}=-\delta_{L-n+1}$ (with $n=2, \ldots, L-1$ ) defined in Eqs. (3.40) and (3.43), respectively. Therefore, for $\xi \gg 1$ one gets

$$
\varepsilon=4 \ln N+2 L[\psi(s)-\psi(2 s)]+\frac{\left(-\psi^{\prime \prime}(s)\right) \pi^{2}}{16 \ln ^{2} N} \sum_{n=2}^{L-1} k_{n}^{2}+\ldots
$$

This expression depends on the set of integers $k_{2}>k_{3}>\ldots$ (with $k_{n}=-k_{L-n+1}$ ) defined in (3.42). Since $\psi^{\prime \prime}(s)<0$ for $s>0$, the minimal value of $\varepsilon$ corresponds to $k_{n}=L+1-2 n$

$$
\varepsilon=4 \ln N+2 L[\psi(s)-\psi(2 s)]+\frac{L^{3}\left(-\psi^{\prime \prime}(s)\right) \pi^{2}}{48 \ln ^{2} N}+\ldots
$$



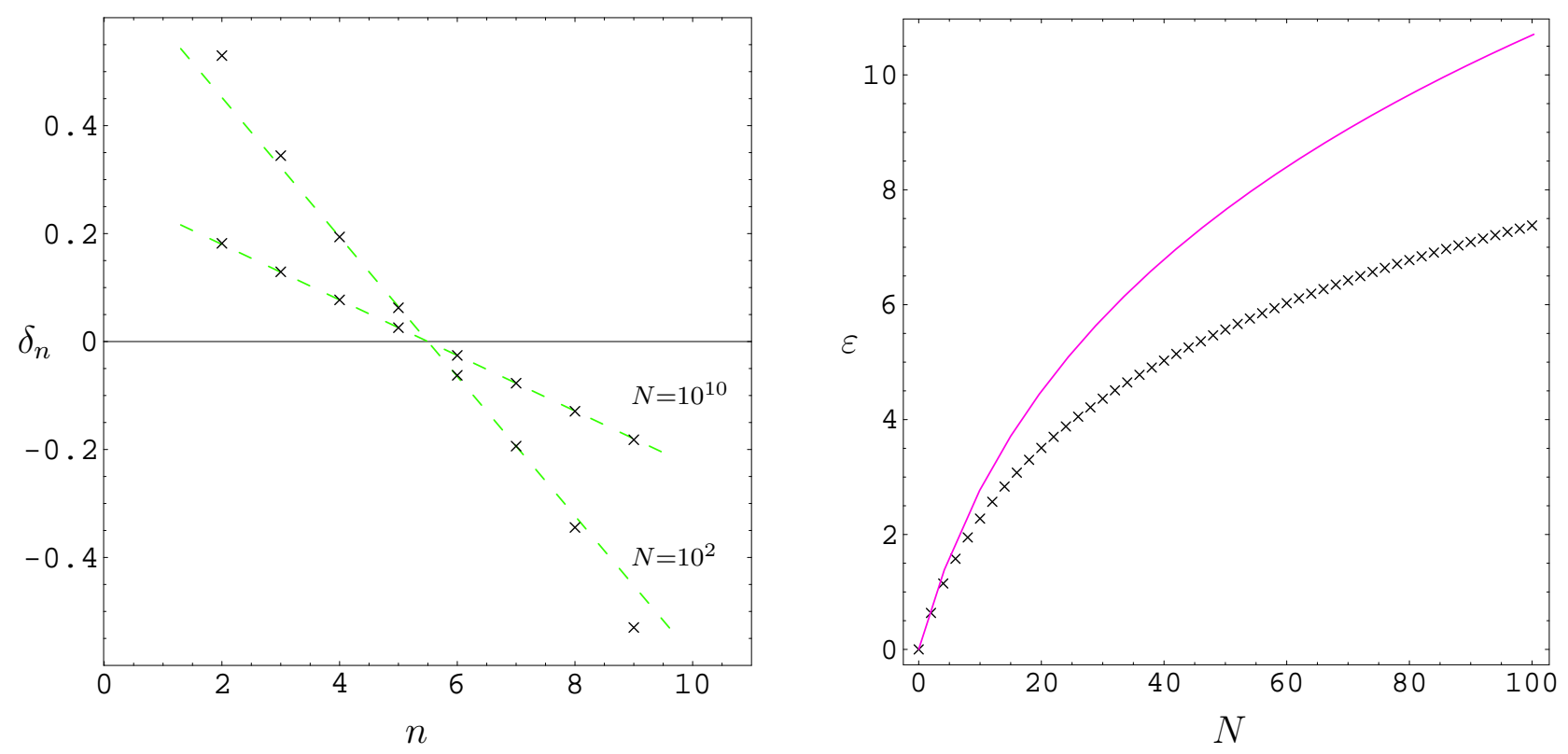

Figure 4: Left panel: "small" roots of the transfer matrix $\delta_{n}$ (with $n=2, \ldots, L-1$ ) for $s=\frac{1}{2}$, $L=10$ and two values of the spin $N=10^{2}$ and $N=10^{10}$. Crosses stand for the solutions to the quantization condition (3.42) and the lines correspond to (3.43) for $k_{n}=L+1-2 n$. The roots tend to approach the line as $\xi=\ln (N / L) / L$ increases from $\xi=0.23$ to $\xi=2.07$. Right panel: the minimal energy for $s=\frac{1}{2}, L=10$ and the total spin $0 \leq N \leq 100$. Crosses denote the exact values, Eq. (2.9), while the solid line stands for the semiclassical expression (3.7). We do not display the data for the asymptotic energy (3.37) with roots deduced from the quantization conditions (3.42), since they are not distinguishable from the exact spectrum.

This relation defines the minimal energy for $\xi \gg 1$. The energy of the excited states is described by (3.48) with another set of $k$-integers but of the same parity. The lowest lying excited state has the same $k$ 's as the minimal energy state except $k_{2}=-k_{L-1}=L-1$. It is separated from the latter by the distance

$$
\Delta \varepsilon=\frac{\pi^{2} L\left(-\psi^{\prime \prime}(s)\right)}{2 \ln ^{2} N} \sim \frac{L}{\ln ^{2} N} .
$$

This relation defines the level spacing in the spectrum of anomalous dimension of the Wilson operators (2.1) close to the minimal anomalous dimension trajectory.

So far our discussion was limited to one loop. Remarkably enough the logarithmic behavior of the anomalous dimension (2.2) persists to all orders of perturbation theory. Higher order corrections to (2.2) merely modify the coefficient in front of $\ln N$ replacing $\lambda$ by an infinite series in the coupling constant,

$$
\gamma(\lambda)=\frac{\lambda}{8 \pi^{2}}\left[4 \ln N+\mathcal{O}\left(N^{0}\right)\right]+\mathcal{O}\left(\lambda^{2}\right)=2 \Gamma_{\text {cusp }}(\lambda) \ln N+\mathcal{O}\left(N^{0}\right),
$$

with $\Gamma_{\text {cusp }}(\lambda)$ being the cusp anomalous dimension. This relation holds true in a generic YangMills theory for arbitrary values of the coupling constant $\lambda$ including the strong coupling regime. In the latter case, one can apply the gauge/string correspondence to obtain a prediction for the cusp anomalous dimension at strong coupling in the $\mathcal{N}=4 \mathrm{SYM}$ theory. In the next section, we 
shall trace the origin of logarithmic scaling of anomalous dimension in the dual picture of folded string rotating on the AdS space.

\section{Classical strings in $\mathrm{AdS}_{3} \times \mathrm{S}^{1}$}

Let us turn to the analysis of anomalous dimensions of composite operators (2.1) on the string side. According to the gauge/string correspondence the Wilson operators are mapped into certain string states whose energy is identified with the scaling dimensions of the former. It is known that in the $\mathcal{N}=4 \mathrm{SYM}$ theory, Wilson operators with the minimal anomalous dimension discussed in the preceding sections are dual to a single-folded string rotating in the $\mathrm{AdS}_{3} \times \mathrm{S}^{1}$ sector of the target space of the type IIB string theory [6, 11].

\subsection{Folded rotating string}

For the folded closed string spinning both in $\mathrm{AdS}_{3}$ and $\mathrm{S}^{1}$, the relevant bosonic part of the superstring action reads

$$
S=-\frac{\sqrt{\lambda}}{2 \pi} \int d^{2} \xi \sqrt{-\operatorname{det}\left\|G_{M N}(X) \partial_{a} X^{M} \partial_{b} X^{N}\right\|},
$$

where the 't Hooft coupling $\lambda=g^{2} N_{c}$ is related to the product of the radius of anti-de Sitter space $R$ and the string tension $1 / \alpha^{\prime}, \sqrt{\lambda}=R^{2} / \alpha^{\prime}$, and the metric in the target space looks like

$$
d s^{2} \equiv G_{M N} d X^{M} d X^{N}=-\cosh ^{2} \rho d t^{2}+\sinh ^{2} \rho d \theta^{2}+d \rho^{2}+d \varphi^{2} .
$$

Here $t, \rho$ are $\theta$ are the global time, the radial coordinate and the angle, respectively, on the $\mathrm{AdS}_{3}$ space and $\varphi$ is the angle on a large circle of $\mathrm{S}^{5}$. The worldsheet coordinates of the string $\xi^{a}=\left(\xi^{0}, \xi^{1}\right)$ are chosen in such a way that

$$
t=\xi^{0}, \quad \theta=\omega \xi^{0}+\xi^{1}, \quad \varphi=\nu \xi^{0}
$$

Here $\omega$ is the angular velocity on AdS and $\nu$ is a boost parameter of the center-of-mass of the folded string on $\mathrm{S}^{5}$. For the rigid folded string the radial variable $\rho$ does not depend on $\xi^{0}$ and is periodic in $\xi^{1}$, that is, $\rho\left(\xi^{1}+2 \pi\right)=\rho\left(\xi^{1}\right)$. The dependence $\rho=\rho\left(\xi^{1}\right)$ is determined by the classical string equations of motion [6, 11, 38. Their solution describes a folded string configuration which is sewed out of four segments running along the radial direction from the center of AdS space, $\rho=0$, towards its boundary by a distance $\rho_{0}$, which depends on the angular velocities entering (4.3)

$$
\operatorname{coth}^{2} \rho_{0}=\frac{\omega^{2}-\nu^{2}}{1-\nu^{2}} \geq 1
$$

The string has two spikes located at $\rho=\rho_{0}$ which are responsible for the logarithmic scaling of the anomalous dimension of Wilson operators at strong coupling [ 8, 38].

Within the gauge/string correspondence, the anomalous dimension of the operators (2.1) is related to the energy $E$ of the classical rotating string as

$$
E=N+L+\gamma(\lambda)
$$


where the Lorentz spin $N$ and twist $L$ are translated into the angular momenta on the $\mathrm{AdS}_{3}$ and $\mathrm{S}^{1}$ spaces, respectively. From the Nambu-Goto action (4.1), one finds these charges as

$$
E=\frac{2 \sqrt{\lambda}}{\pi} \frac{\sqrt{-\chi}}{\sqrt{1-\nu^{2}}} \mathbb{E}(\chi), \quad N=\frac{2 \sqrt{\lambda}}{\pi} \frac{\omega \sqrt{-\chi}}{\sqrt{1-\nu^{2}}}[\mathbb{E}(\chi)-\mathbb{K}(\chi)], \quad L=\frac{2 \sqrt{\lambda}}{\pi} \frac{\nu \sqrt{-\chi}}{\sqrt{1-\nu^{2}}} \mathbb{K}(\chi),
$$

where $\mathbb{K}(\chi)$ and $\mathbb{E}(\chi)$ are the elliptic functions of the first and second kind, respectively, and the auxiliary (negative valued) parameter $\chi$ is related to the distance $\rho_{0}$ from the center of anti-de Sitter space to the end of the string extending to its boundary,

$$
\chi=-\sinh ^{2} \rho_{0} .
$$

Excluding $\omega$ and $\nu$ in favor of $\chi$, Eqs. (4.6) can be cast into the parametric form [18]

$$
\left(\frac{E}{\mathbb{E}(\chi)}\right)^{2}-\left(\frac{L}{\mathbb{K}(\chi)}\right)^{2}=-\frac{4 \lambda}{\pi^{2}} \chi, \quad\left(\frac{N}{\mathbb{E}(\chi)-\mathbb{K}(\chi)}\right)^{2}-\left(\frac{L}{\mathbb{K}(\chi)}\right)^{2}=\frac{4 \lambda}{\pi^{2}}(1-\chi) .
$$

Using these relations, one can analyze the anomalous dimension $\gamma=E-N-L$ at strong coupling in the semiclassical limit of large angular momenta $L, N \rightarrow \infty$ with $N / \sqrt{\lambda}$ and $L / \sqrt{\lambda}$ kept fixed. In this way one finds from (4.8) that the two limiting cases $N \ll L$ and $N \gg L$ correspond to the short and long strings, $\rho_{0} \ll 1$ and $\rho_{0} \gg 1$, respectively.

It is well-known [6, 11] that in the short string limit, $\rho_{0} \ll 1$, or equivalently $(-\chi) \ll 1$, the anomalous dimension exhibits the BMN scaling

$$
\gamma(\lambda)=L\left[\lambda^{\prime} \gamma^{(0)}+\left(\lambda^{\prime}\right)^{2} \gamma^{(1)}+\ldots\right]
$$

with $\gamma^{(0)}, \gamma^{(1)}, \ldots$ being functions of the ratio $N / L$ and $\lambda^{\prime}=\lambda /(\pi L)^{2}$ determining the BMN coupling constant. Substituting $\chi=\chi_{0}+\lambda^{\prime} \chi_{1}+\ldots$ into (4.8) and matching the coefficients in the expansion of both sides of (4.8) in powers of $\lambda^{\prime}$, one finds for $N / L \ll 1$ [10, 11, 18]

$$
\gamma^{(0)}=\frac{\pi^{2}}{2} \frac{N}{L}+\ldots, \quad \gamma^{(1)}=-\frac{\pi^{4}}{8} \frac{N}{L}+\ldots
$$

The lowest order term in (4.9) matches the one-loop result on the gauge theory side, Eq. (3.12), for $s=\frac{1}{2}$ and $m=1$

$$
\gamma^{(0)}=\frac{L}{8} \varepsilon .
$$

In a similar manner, in the long string limit, $\rho_{0} \rightarrow \infty$, or equivalently $(-\chi) \rightarrow \infty$, assuming that $\chi$ has a regular expansion in powers of $\lambda^{\prime}$, one finds from Eq. (4.8) that the leading order parameter $\chi_{0}$ scales for $N / L \gg 1$ as

$$
\chi_{0}=-\frac{N}{2 L} \ln \frac{N}{L} .
$$

This leads to the following expressions for the coefficients of the BMN expansion of the anomalous dimension (4.9) for $N / L \gg 1$

$$
\begin{aligned}
& \gamma^{(0)}=\frac{1}{2} \ln ^{2}\left(-\chi_{0}\right)+\ldots=\frac{1}{2} \ln ^{2}(N / L)+\ldots, \\
& \gamma^{(1)}=-\frac{1}{8} \ln ^{4}\left(-\chi_{0}\right)+\ldots=-\frac{1}{8} \ln ^{4}(N / L)+\ldots,
\end{aligned}
$$


where ellipses denote terms subleading for $\left(-\chi_{0}\right) \rightarrow \infty$. In agreement with (4.11), the relation (4.13) matches the one-loop expression for the anomalous dimension on the gauge theory side, Eq. (3.14). Moreover, one can show [18] that the relation (4.11) (upon the Landen transformation defined below in Eq. (4.33) ) holds for an arbitrary value of $N / L$ with $\gamma^{(0)}$ given by (4.8) and (4.9) and $\varepsilon$ defined in (3.7) and (3.4).

So far we see no trace of expected scaling of the anomalous dimension $\gamma(\lambda) \sim \ln N$ for $N \gg L$. We recall that on the gauge theory side the semiclassical expansion for the one-loop energy $\varepsilon$ was divergent for $N \gg L$ and in order to recover the logarithmic scaling $\varepsilon \sim \ln N$ we had to resum the entire semiclassical series in powers of the parameter $\xi=\ln (N / L) / L$. It turns out that a similar phenomenon happens for the series (4.9) on the string side although the parameter of the semiclassical expansion is different and equals

$$
\xi_{\text {str }}=\frac{\lambda}{L^{2}} \ln ^{2} \frac{N}{L}=\lambda \xi^{2} .
$$

It can be easily identified by comparing the contribution to (4.9) from $\gamma^{(0)}$ and $\gamma^{(1)}$, Eq. (4.13).

To sum up the infinite series in (4.9) for $\xi_{\text {str }} \gg 1$ we return to (4.8) and rewrite the first relation as

$$
\gamma(\lambda)=L\left[\sqrt{1-4 \lambda^{\prime} \chi \mathbb{K}^{2}(\chi)}-1+\Delta \gamma\right]
$$

with $\lambda^{\prime}=\lambda /(\pi L)^{2}$ and

$$
\Delta \gamma=-\frac{4 \lambda^{\prime} \mathbb{K}(\chi)[\mathbb{E}(\chi)-\mathbb{K}(\chi)]}{\sqrt{1-4 \lambda^{\prime} \chi \mathbb{K}^{2}(\chi)}+\sqrt{1-4 \lambda^{\prime}(\chi-1) \mathbb{K}^{2}(\chi)}} .
$$

In the limit of a long string, for $(-\chi) \rightarrow \infty$, the expression for $\gamma(\lambda)$ can be expanded in powers of $4 \lambda^{\prime}(-\chi) \mathbb{K}^{2}(\chi) \sim \lambda^{\prime} \ln ^{2}(-\chi)$. Examining the expression for $\Delta \gamma$ one finds that its contribution to $\gamma(\lambda)$ is suppressed by the factor $[\mathbb{E}(\chi)-\mathbb{K}(\chi)] /(-\chi \mathbb{K}(\chi)) \sim 1 / \ln (-\chi)$ compared to the first term in the square brackets in (4.15). This implies that in the limit $(-\chi) \rightarrow \infty$ with $\lambda^{\prime} \ln ^{2}(-\chi)=$ fixed the leading asymptotic behavior of (4.15) reads

$$
\gamma(\lambda)=L\left[\sqrt{1+\lambda^{\prime} \ln ^{2}(-\chi)}-1\right]+\ldots
$$

This relation resums all double-logarithmic corrections $\sim L\left[\lambda^{\prime} \ln ^{2}(-\chi)\right]^{n}$ to the anomalous dimension $\gamma(\lambda)$ to all orders $n$ at strong coupling. In particular, for $n=1$ and $n=2$ it reproduces (4.13). For $\lambda^{\prime} \ln ^{2}(-\chi) \ll 1$ one expands the square-root in (4.17) and arrives at a BMN-like expansion (4.9). At the same time, for $\lambda^{\prime} \ln ^{2}(-\chi) \gg 1$ the relation (4.17) leads to the expression

$$
\gamma(\lambda)=L \sqrt{\lambda^{\prime}} \ln (-\chi)+\ldots=\frac{\sqrt{\lambda}}{\pi} \ln (-\chi)+\ldots
$$

For $(-\chi) \rightarrow \infty$, the dependence of $\chi$ on the ratio $N / L$ and the BMN coupling $\lambda^{\prime}$ follows from the second relation in (4.8)

$$
\frac{1}{4 \chi^{2}}=\frac{L^{2}}{N^{2}}\left[\frac{1}{\ln ^{2}(-\chi)}+\lambda^{\prime}\right] .
$$

For $\xi_{\text {srt }}<1$ and $N \gg L$ one finds from (4.19) and (4.14) that $\chi \sim \chi_{0}=-\frac{N}{2 L} \ln \frac{N}{L}$ and, therefore,

$$
\gamma(\lambda)=L\left[\sqrt{1+\frac{\lambda}{(\pi L)^{2}} \ln ^{2}(N / L)}-1\right]+\ldots
$$


For $\xi_{\text {srt }} \gg 1$ one finds from (4.19) and (4.14) that $(-\chi) \sim N /\left(2 L \sqrt{\lambda^{\prime}}\right) \sim N / \sqrt{\lambda}$ and, therefore,

$$
\gamma(\lambda)=\frac{\sqrt{\lambda}}{\pi} \ln (N / \sqrt{\lambda})+\ldots
$$

This relation reproduces the correct asymptotic behavior of the anomalous dimension in the regime (1.4) and it is in agreement with the results of Refs. [6, 11, 38,

The coefficient in front of $\ln N$ in (4.21) determines the leading asymptotics of the cusp anomalous dimension in the strong coupling regime in the $\mathcal{N}=4 \mathrm{SYM}$ theory, Eqs. (1.1) and (1.2). It follows from the relations (4.20) and (4.21) that quite remarkably the cusp anomaly at strong coupling can be obtained by resumming double logarithmic terms $\sim L\left[\lambda \ln ^{2}(N / L) / L^{2}\right]^{k}$ in the expansion of anomalous dimensions of operators of higher twist $L$ and large Lorentz spin $N \gg L$.

\subsection{Two-cut solution in string sigma model}

In the previous section, we observed that for $N \gg L$ the dependence of the anomalous dimension $\gamma(\lambda)$ on the coupling constant is different for $\xi_{\text {str }}<1$ and $\xi_{\text {str }} \gg 1$. In the former case, $\gamma(\lambda)$ has a BMN-like expansion in powers of $\lambda / L^{2}$, while in the latter case $\gamma(\lambda)$ is not analytical in $\lambda$ and scales logarithmically $\sim \sqrt{\lambda} \ln N$. The reason for this non-analyticity is that for $\xi_{\text {str }} \gg 1$ the end-points of the rotating string approach the boundary of the AdS space and the dominant contribution to the energy of the string, or equivalently the anomalous dimension (4.5), comes from the vicinity of two spikes, $\gamma(\lambda) \sim(\sqrt{\lambda} / \pi) \cdot 2 \rho_{0}$. Here the radial coordinate of the spikes scales for $\xi_{\text {str }} \gg 1$ as $\rho_{0} \sim \frac{1}{2} \ln (-\chi) \sim \frac{1}{2} \ln (N / \sqrt{\lambda})$, Eqs. (4.7) and (4.19). The phenomenon is rather general [8, 38] and it holds true for classical string configurations with an arbitrary number of spikes $n$. In that case, each spike provides a logarithmic contribution to the energy and the anomalous dimension scales for $\xi_{\text {str }} \gg 1$ as $\gamma(\lambda)=n \sqrt{\lambda} /(2 \pi) \ln (N / \sqrt{\lambda})$. At first glance, this mechanism is rather different from the one in gauge theory. We recall that in gauge theory, to one-loop order, the logarithmic scaling of anomalous dimension for $N \gg L$ arises due to collision of cuts for the spectral curve of the spin chain, Eq. (2.19). It is known that the classical equations of motion for the string on the $\mathrm{AdS}_{5} \times \mathrm{S}^{5}$ background are completely integrable [22, 21] and their solutions are parameterized by the spectral curves. Moreover, for the strings on the $\operatorname{Ad} \mathrm{S}_{3} \times \mathrm{S}^{1}$ part of the target space the spectral curve can be identified as a complex hyperelliptic curve [23]. For the folded rotating closed string discussed in the previous section, it is given by the elliptic curve with symmetric branching points on the real axis [24]

$$
\Gamma_{\mathrm{str}}: \quad y^{2}=\left(x^{2}-a_{\mathrm{str}}^{2}\right)\left(x^{2}-b_{\mathrm{str}}^{2}\right)
$$

with $a_{\text {str }}$ and $b_{\text {str }}$ taking positive values, $b_{\text {str }}<a_{\text {str }}$. In this section, we shall translate different asymptotic behavior of the anomalous dimensions for $N \gg L$, Eqs. (4.20) and (4.21), into properties of the curve (4.22) and reveal the mechanism responsible for the logarithmic scaling (4.21).

Similar to the classical $S L(2)$ spin chain, the classical string equations of motion admit the Lax representation and they can be solved exactly by constructing the Baker-Akhiezer function [23, 22. As before, the Bloch-Floquet multiplier for this function gives rise to the (quasi)momentum $p(x)$ which is the generating function for the conserved charge including the energy. For the folded rotating string configuration, $p^{\prime}(x)$ is an analytical function in the complex plane with 
two symmetric cuts $\left[-a_{\mathrm{str}},-b_{\mathrm{str}}\right] \cup\left[b_{\mathrm{str}}, a_{\mathrm{str}}\right]$. It is uniquely fixed by the requirement that $d p=$ $p^{\prime}(x) d x$ should be a meromorphic differential on the complex curve (4.22) satisfying the following conditions on the upper sheet of $\Gamma_{\text {str }}[24$ :

- Prescribed asymptotics at infinity and at the origin

$$
d p \stackrel{x \rightarrow \infty}{\sim}-2 \frac{d x}{x^{2}} \frac{E+N}{L}, \quad d p \stackrel{x \rightarrow 0}{\sim}-d x \frac{2}{\lambda^{\prime}} \frac{E-N}{L}
$$

- Single-valuedness condition

$$
\int_{b_{\mathrm{str}}}^{a_{\mathrm{str}}} d p=0, \quad \int_{a_{\mathrm{str}}}^{\infty} d p=-\pi m
$$

- Double poles at $x= \pm \sqrt{\lambda} /(\pi L) \equiv \sqrt{\lambda^{\prime}}$

$$
d p \sim d x\left[-\frac{1}{\left(x \pm \sqrt{\lambda^{\prime}}\right)^{2}}+\mathcal{O}\left(\left(x \pm \sqrt{\lambda^{\prime}}\right)^{0}\right)\right]
$$

The resulting expression for the differential $d p$ takes the form

$$
d p=\frac{d x}{y}\left[\frac{y_{+}}{\left(x-\sqrt{\lambda^{\prime}}\right)^{2}}+\frac{y_{+}^{\prime}}{x-\sqrt{\lambda^{\prime}}}+\frac{y_{+}}{\left(x+\sqrt{\lambda^{\prime}}\right)^{2}}-\frac{y_{+}^{\prime}}{x+\sqrt{\lambda^{\prime}}}+C\right]
$$

where $y=y(x)$ is defined in (4.22), $y_{+}=y\left(\sqrt{\lambda^{\prime}}\right)$ and $y_{+}^{\prime}=y^{\prime}\left(\sqrt{\lambda^{\prime}}\right)$.

Equation (4.26) depends on three parameters $a_{\text {str }}, b_{\text {str }}$ and $C$. They are fixed by the normalization conditions (4.23) and (4.24) as

$$
\begin{aligned}
b_{\mathrm{str}} & =\frac{1}{m \mathbb{K}(\tau)}\left[\left(1-\frac{\lambda^{\prime}}{a_{\mathrm{str}}^{2}}\right)\left(1-\frac{\lambda^{\prime}}{b_{\mathrm{str}}^{2}}\right)\right]^{-1 / 2} \\
C & =-\frac{m a_{\mathrm{str}}}{2}\left[\mathbb{E}(\tau)-\frac{\lambda^{\prime}}{a_{\mathrm{str}}^{2}} \mathbb{K}(\tau)\right],
\end{aligned}
$$

with the modular parameter $\tau=1-b_{\text {str }}^{2} / a_{\text {str }}^{2}$. In addition, one finds from (4.23) the following expressions for the ratio $N / L$ and for the anomalous dimension $\gamma(\lambda)=E-N-L$

$$
\begin{aligned}
N / L & =\frac{m}{2}\left[\mathbb{E}(\tau)\left(a_{\text {str }}+\frac{\lambda^{\prime}}{b_{\text {str }}}\right)-\mathbb{K}(\tau)\left(b_{\text {str }}+\frac{\lambda^{\prime}}{a_{\text {str }}}\right)\right], \\
\gamma(\lambda) / L & =m\left[\mathbb{K}(\tau) b_{\text {str }}-\mathbb{E}(\tau) \frac{\lambda^{\prime}}{b_{\text {str }}}\right]-1,
\end{aligned}
$$

with $b_{\text {str }}$ defined in (4.27) and $a_{\text {str }}=b_{\text {str }} / \sqrt{1-\tau}$.

Let us examine the dependence of the anomalous dimension on the coupling constant $\lambda^{\prime}=$ $\lambda /(\pi L)^{2}$. Assuming that $b_{\text {str }}$ and $\tau$ both admit a regular expansion in powers of $\lambda^{\prime}$, one substitutes into (4.27) and (4.28)

$$
b_{\mathrm{str}}=b_{\mathrm{str}}^{(0)}+\lambda^{\prime} b_{\mathrm{str}}^{(1)}++\ldots, \quad \tau=\tau^{(0)}+\lambda^{\prime} \tau^{(1)}+\ldots,
$$


and matches the coefficients in front of powers of $\lambda^{\prime}$. In this way, one obtains to leading order

$$
b_{\mathrm{str}}^{(0)}=\frac{1}{m \mathbb{K}\left(\tau^{(0)}\right)}, \quad \frac{N}{L}=\frac{1}{2}\left[\frac{\mathbb{E}\left(\tau^{(0)}\right)}{\sqrt{1-\tau^{(0)}} \mathbb{K}\left(\tau^{(0)}\right)}-1\right],
$$

and all subleading corrections to (4.29) are expressed in terms of the leading terms. Then, the first few coefficients of the BMN series for anomalous dimension (4.9) are given by

$$
\begin{aligned}
& \gamma^{(0)}=\frac{m^{2}}{2} \mathbb{K}\left(\tau^{(0)}\right)\left[\left(2-\tau^{(0)}\right) \mathbb{K}\left(\tau^{(0)}\right)-2 \mathbb{E}\left(\tau^{(0)}\right)\right] \\
& \gamma^{(1)}=\frac{m^{4}}{8} \mathbb{K}^{3}\left(\tau^{(0)}\right)\left[\left(4\left(2-\tau^{(0)}\right) \sqrt{1-\tau^{(0)}}-\left(\tau^{(0)}\right)^{2}\right) \mathbb{K}\left(\tau^{(0)}\right)-8 \sqrt{1-\tau^{(0)}} \mathbb{E}\left(\tau^{(0)}\right)\right]
\end{aligned}
$$

Together with the second relation in (4.30), they define the parametric dependence of the anomalous dimension (4.9) on $N / L$.

Equations (4.30) determine perturbative corrections to the anomalous dimension of long scalar operators in the $\mathcal{N}=4 \mathrm{SYM}$ theory. According to (4.11), it should match at one-loop order a similar asymptotic expression (3.7) obtained on the gauge theory side within the semiclassical approach. The conformal spin of scalars equals $s=1 / 2$ and the scaling parameter $\beta=L /(L+$ $2 N)$, Eq. (2.10), is given by $\beta=\sqrt{1-\tau^{(0)}} \mathbb{K}\left(\tau^{(0)}\right) / \mathbb{E}\left(\tau^{(0)}\right)$ in agreement with (3.4). Then, one observes that $\gamma^{(0)}$ and $\varepsilon$ given by (4.31) and (3.7), respectively, verify the relation (4.11). The expressions for the branching points $b_{\mathrm{str}}^{(0)}$ and $b$, defined in (4.30) and (3.4), respectively, are different, $b / b_{\mathrm{str}}^{(0)}=\beta / 2$, but the agreement can be restored through the rescaling of the local complex parameter $x$ in the definition of the curve (4.22), $x \rightarrow x \beta / 2$.

The functional form of the anomalous dimensions (4.28) is different compared to the ones found in the previous section, Eq. (4.8). The agreement is achieved by means of the Landen transformation of the modular parameters [18]

$$
\chi^{(0)}=-\frac{\left(1-\sqrt{1-\tau^{(0)}}\right)^{2}}{4 \sqrt{1-\tau^{(0)}}},
$$

upon which the relations (4.28) and (4.8) coincide provided that $m=1$. Remember that $(-\chi)$ depends on the radial coordinate of the spike $\rho_{0}$, Eq. (4.7), so that $\sqrt{1-\tau}=\mathrm{e}^{-2 \rho_{0}}$ and the long string limit $\rho_{0} \rightarrow \infty$ corresponds to $\tau \rightarrow 1$. We have demonstrated in Sect. 2.3 that on the gauge theory side the limit $\tau \rightarrow 1$ corresponds to $a \rightarrow 1 /(2 m)$ and $b \rightarrow 0$, Eq. (3.13). As a consequence, the two cuts $[-a,-b]$ and $[b, a]$ collide at the origin yielding the logarithmic scaling of the one-loop anomalous dimension (2.42). Let us examine the limit $\tau \rightarrow 1$ of the obtained stringy expressions (4.27) and (4.28).

Since $\mathbb{K}(\tau) \sim-\frac{1}{2} \ln (1-\tau)$ for $\tau \rightarrow 1$, one would expect from (4.27) that $b_{\text {str }}$ should vanish in this limit. Indeed, this is the case for $\lambda^{\prime}=0$ while for $\lambda^{\prime} \neq 0$ one deduces from (4.27) that the reality condition for $b_{\text {str }}$ implies that its minimal value is bounded as $b_{\text {str }} \geq \sqrt{\lambda^{\prime}}$. Carefully examining (4.27) for $\tau \rightarrow 1$ one finds

$$
b_{\mathrm{str}}=\sqrt{\lambda^{\prime}+\frac{1}{m^{2} \ln ^{2} \sqrt{1-\tau}}}
$$

For $\lambda^{\prime}=0$ this relation coincides with the one-loop expression $b_{\mathrm{str}}^{(0)}=2 b / \beta$, Eq. (3.13). Matching (4.34) into (4.29) we conclude that higher order corrections to $b_{\text {str }}$ push its minimal possible value 
away from the origin and, therefore, prevent the two cuts $\left[-a_{\mathrm{str}},-b_{\mathrm{str}}\right]$ and $\left[b_{\mathrm{str}}, a_{\mathrm{str}}\right]$ to collide. From (4.28) one finds in the limit $\tau \rightarrow 1$

$$
\beta \approx \frac{L}{2 N}=\frac{\sqrt{1-\tau}}{m b_{\text {str }}}+\ldots, \quad \gamma(\lambda)=L\left[-m b_{\text {str }} \ln \sqrt{1-\tau}-1\right]+\ldots
$$

The asymptotic behavior of these expressions depends on the value of the parameter $\xi_{\text {str }}=$ $\lambda^{\prime} \ln ^{2}(N / L)$, Eq. (4.14).

For $\xi_{\text {str }}<1$ the expression for $b_{\text {str }}$, Eq. (4.34), admits a series expansion in $\lambda^{\prime}$ and leads together with (4.35) to

$$
b_{\mathrm{str}}=\frac{1}{m \ln \frac{N}{L}}+\ldots, \quad a_{\mathrm{str}}=\frac{N}{2 m L}+\ldots, \quad \sqrt{1-\tau}=\frac{L / N}{2 \ln \frac{N}{L}}+\ldots .
$$

Substitution of (4.34) into (4.35) yields an expression for the anomalous dimension $\gamma(\lambda)$ which coincides with (4.20) for $m=1$. It is instructive to compare the positions of the cuts in gauge theory, $a$ and $b$, and on the string side, $\widehat{a}=\beta a_{\text {str }} / 2$ and $\widehat{b}=\beta b_{\text {str }} / 2$. Here the additional factor $\beta / 2$ appears due to a different definition of the local parameter $x$ on the spectral curves (2.37) and (4.22). In this way we find $\widehat{a}=1 /(2 m)+\ldots$ and $\widehat{b}=(L / N) /(4 m \ln (N / L))+\ldots$ which coincides with the similar relation (3.13) in gauge theory to one-loop order.

For $\xi_{\text {str }} \gg 1$ the expression for $b_{\text {str }}$, Eq. (4.34), is not analytical in the BMN coupling $\lambda^{\prime}=$ $\lambda /(\pi L)^{2}$

$$
b_{\mathrm{str}}=\sqrt{\lambda^{\prime}}+\ldots, \quad a_{\mathrm{str}}=\frac{N}{2 m L}+\ldots, \quad \sqrt{1-\tau}=\frac{L m}{2 N} \sqrt{\lambda^{\prime}}+\ldots
$$

while the expression for $a_{\text {str }}$ is the same as in (4.36). This suggests that for $\xi_{\text {str }} \gg 1$ higher order corrections only modify the lower edge of the cut. One finds from (4.35) that the anomalous dimension scales as $\gamma(\lambda) \sim m \sqrt{\lambda} \ln (N /(m \sqrt{\lambda}))$ and matches (4.21) for $m=1$. We conclude that the logarithmic scaling of the anomalous dimension for $N \gg L$ on the string side is realized when

the inner boundary of the cut $b_{\text {str }}$ approaches its minimal possible value $\sqrt{\lambda^{\prime}}$ (see Fig. 2 ) which coincides with the position of the double pole of the differential (4.25).

\section{Conclusion}

In the present paper we have studied the properties of anomalous dimensions of Wilson operators of higher twist $L$ and large Lorentz spin $N$ in the weak and strong coupling regimes by making use of the remarkable integrability symmetry on both sides of the gauge/string correspondence. We concentrated on operators which have the minimal anomalous dimension for given Lorentz spin and put a special emphasis on the appearance of the single-logarithmic behavior, $\gamma(\lambda) \sim \ln N$, in the thermodynamic limit $L \rightarrow \infty$.

On the gauge theory side, we applied the method of the Baxter $Q$-operator to identify different regimes of the minimal anomalous dimension in integrable sectors of (supersymmetric) Yang-Mills theory to one-loop order. We argued that for $N \gg L$ the asymptotic behavior of $\gamma(\lambda)$ is controlled by the parameter $\xi=\ln (N / L) / L$. For $\xi<1$ the anomalous dimension possesses the BMN scaling $\gamma(\lambda) \sim \lambda / L$, while for $\xi \gg 1$ it scales logarithmically with $N$. Transition to the second, logarithmic regime manifests itself through the divergence of the semiclassical expansion 
for $\gamma(\lambda)$ as $\xi \sim 1$. The anomalous dimension is uniquely determined by the configuration of Bethe roots which condense in the thermodynamic limit on two symmetric cuts. We demonstrated that the semiclassical approach breaks down for $\xi \sim 1$ due to the collision of cuts at the origin and worked out an asymptotic expression for anomalous dimensions which is valid throughout the entire region of $\xi$.

On the string theory side, we used the identification of the minimal anomalous dimension of scalar operators in the $\mathcal{N}=4 \mathrm{SYM}$ at strong coupling as the energy of folded string rotating on $\mathrm{AdS}_{3} \times \mathrm{S}^{1}$ part of the target space. Similar to the previous case, the anomalous dimension has different behavior for $N \gg L$ depending on the value of the parameter $\xi_{\text {str }}=\lambda \ln ^{2}(N / L) / L^{2}$. For $\xi_{\text {str }}<1$ the anomalous dimension has a regular expansion in powers of the BMN coupling and its lowest term matches the one-loop expression for $\gamma(\lambda)$ at weak coupling for $\xi<1$. For $\xi_{\text {str }} \gg 1$ the anomalous dimension scales logarithmically but its dependence on the 't Hooft coupling is not analytical anymore. We described the latter regime using two different (although equivalent) configurations. In terms of the folded rotating string the logarithmic scaling occurs when two most distant points of the string (two spikes) approach the boundary of the AdS space. In terms of the spectral curve for the classical string sigma model, the same configuration is described by the elliptic curve with symmetric branching points. Different regimes of $\gamma(\lambda)$ arise depending on the position of the branching points. In the logarithmic regime, the inner branching points approach the minimal possible value $\pm \sqrt{\lambda^{\prime}}$ so that the anomalous dimension ceases to obey the BMN scaling.

Integrability played a key role in our analysis. In generic (supersymmetric) Yang-Mills theory it holds to one-loop order for Wilson operators belonging to special, holomorphic sectors only. We would like to stress that logarithmic behavior of anomalous dimensions is not tied to integrability. In non-integrable sectors the mixing matrix for Wilson operators contains additional terms which break integrability symmetry. They do not affect however the logarithmic scaling of anomalous dimension for $N \rightarrow \infty$. The reason for this is that the logarithmic scaling of anomalous dimension can be associated with the contribution of soft gluons (i.e., gauge field quanta). Soft gluon radiation is not sensitive to the quantum numbers of Wilson operators (except of the total Lorentz spin) and, therefore, it provides the same logarithmic contribution to the anomalous dimension of Wilson operators in all sectors. Integrability allows one to identify various regimes of the asymptotic behavior of anomalous dimensions and to determine the "critical" value of $\ln (N / L) \sim L$ at which the logarithmic scaling sets in.

As a function of the total Lorentz spin $N$, the anomalous dimensions of twist- $L$ operators occupy a band. Our discussion was restricted to the minimal anomalous dimensions belonging to the lower edge of the band. It would be interesting to extend the above analysis to excited states (3.47) with $m>1$ and describe the band structure occupied by the anomalous dimensions as it arises form the Baxter equation on the gauge theory side. In string theory, there are two different classical configurations yielding the same logarithmic asymptotics of anomalous dimensions at strong coupling — the multiple folded string [11] and the spiky string [38. The coefficient in front of the logarithm, $m$, is twice the number of foldings of the string on itself, in the former case and it is the number of spikes, in the latter one. It would be interesting to construct a generic configuration which interpolates between both solutions.

Note added: After the work has been completed we were informed by Yuji Satoh that he, in collaboration with Kazuhiro Sakai, analyzed the spectrum of anomalous dimensions with a special emphasis on the appearance of logarithmic scaling in the large spin limit. For the two- 
cut solution, their findings are in agreement with the analysis presented in sections 3.1$]$ and 4.2 of this paper. In particular, they also came to the conclusion that, in the thermodynamical limit $L \rightarrow \infty$, the semiclassical approach to the Bethe Ansatz equations is only applicable for $\ln (N / L)<L$ and one can not reproduce $\sim \ln N$ behavior of anomalous dimensions unless the finite size corrections in $1 / L$ are included.

We would like to thank to thank S. Derkachov, Yu. Makeenko, A. Manashov, Y. Satoh, F. Smirnov, E. Sokatchev, B. Stefanski and A. Tseytlin for very useful discussions and correspondence. A.G. is grateful to Laboratoire de Physique Théorique (Orsay) for hospitality extended to him during his stay which was partially supported by the Russian-French Exchange Program. This work was supported by the U.S. National Science Foundation under grant no. PHY-0456520 (A.B.), by the grant CRDF-RUP2-261-MO-04 from the U.S. Civilian Research and Development Foundation and Russian Foundation for Basic Research under contract RFBR-04011-00646 (A.G.).

\section{Appendix Asymptotic expression for the energy}

The asymptotic expression for the energy (3.37) involves roots of the transfer matrix and is not well suited for performing the thermodynamical limit $L \rightarrow \infty$. Let us rewrite the energy in terms of the transfer matrix itself. We notice from (3.30) that

$$
\frac{d}{d u} \ln t_{L}(u)=\sum_{k=1}^{L} \frac{1}{u-\delta_{k}}
$$

and replace the $\psi$-functions in (3.37) by series representation

$$
\psi\left(s+i \delta_{k}\right)+\psi\left(s-i \delta_{k}\right)-2 \psi(2 s)=\sum_{k=0}^{\infty}\left[\frac{i}{i(s+k)-\delta_{k}}+\frac{-i}{-i(s+k)-\delta_{k}}-\frac{2}{2 s+k}\right] .
$$

Then, the energy (3.37) can be written as an infinite sum

$$
E=2 \ln 2-\sum_{k=0}^{\infty} \frac{d}{d k} f(k), \quad f(x)=\ln \left[\frac{t_{L}(i(s+x)) t_{L}(-i(s+x))}{(2 s+x)^{2 L}}\right]
$$

where $f(x) \rightarrow 2 \ln 2$ for $x \rightarrow \infty$. The sum can be evaluated with a help of the Euler-Maclaurin summation formula

$$
E=f(0)-\frac{1}{2} f^{\prime}(0)+\sum_{k=1}^{\infty} \frac{B_{2 k}}{(2 k) !} f^{(2 k)}(0)=\frac{\mathrm{d}}{\mathrm{e}^{\mathrm{d}}-1} f(0)
$$

with $B_{2 k}$ being Bernoulli numbers. Taking $u= \pm i(s+x)$ in the Baxter equation (2.3), one obtains $t_{L}( \pm i(s+x))$ as the ratio of $Q$-functions and finds for $x \rightarrow 0$

$$
f(x)=\ln \left[\frac{Q(i(s+x+1))}{Q(i(s+x))} \frac{Q(-i(s+x+1))}{Q(-i(s+x))}\right]+\mathcal{O}\left(x^{L}\right) .
$$


Then, one uses the WKB ansatz $Q(u) \sim \exp \left(\eta^{-1} S(u \eta)\right)$ to get

$$
f(x)=\frac{1}{\eta}[S(i(s+x+1) \eta)-S(i(s+x) \eta)+S(-i(s+x+1) \eta)-S(-i(s+x) \eta)]+\mathcal{O}\left(x^{L}\right) .
$$

Substituting this expression into (A.4) one finally obtains the relation

$$
E=f(0)-\frac{1}{2} f^{\prime}(0)+\ldots=-2 s \eta S^{\prime \prime}(0)+\ldots=-\frac{2 \beta}{L} S^{\prime \prime}(0)+\ldots
$$

which coincides with the semiclassical expression (2.33). It is important to keep in mind that Eq. (A.7) was obtained under the assumption that contribution of terms with higher derivatives is small, $f^{\prime \prime}(0) \ll f^{\prime}(0)$, or equivalently $\eta S^{\prime \prime \prime}(0) \ll S^{\prime \prime}(0)$. This relation is not satisfied if the distribution of Bethe roots scales at the origin as $\sim \ln x$, Eq. (2.40). In that case, one has to rely on the formula (A.3) which resums all singular terms.

\section{References}

[1] J.C. Collins, Adv. Ser. Direct. High Ener. Phys. 5 (1989) 573.

[2] G.P. Korchemsky, Mod. Phys. Lett. A 4 (1989) 1257;

G.P. Korchemsky, G. Marchesini, Nucl. Phys. B 406 (1993) 225.

[3] A.M. Polyakov, Nucl. Phys. B 164 (1980) 171.

[4] S. Moch, J.A.M. Vermaseren, A. Vogt, Nucl. Phys. B 688 (2004) 101.

[5] Z. Bern, L.J. Dixon, V.A. Smirnov, Phys. Rev. D 72 (2005) 085001.

[6] S.S. Gubser, I.R. Klebanov, A.M. Polyakov, Nucl. Phys. B 636 (2002) 99.

[7] M. Kruczenski, J. High Ener. Phys. 0212 (2002) 024;

Yu. Makeenko, J. High Ener. Phys. 0301 (2003) 007.

[8] A.V. Belitsky, A.S. Gorsky, G.P. Korchemsky, Nucl. Phys. B 667 (2003) 3.

[9] J.M. Maldacena, Adv. Theor. Math. Phys. 2 (1998) 231;

S.S. Gubser, I.R. Klebanov, A.M. Polyakov, Phys. Lett. B 428 (1998) 105;

E. Witten, Adv. Theor. Math. Phys. 2 (1998) 253.

[10] D. Berenstein, J.M. Maldacena, H. Nastase, J. High Ener. Phys. 0204 (2002) 013.

[11] S. Frolov, A.A. Tseytlin, J. High Ener. Phys. 0206 (2002) 007.

[12] A.A. Tseytlin, in Ian Kogan Memorial Volume, From Fields to Stings: Circumnavigating Theoretical Physics, eds. M. Shifman, A. Vainshtein, J. Wheater, World Scientific (Singapore, 2004) vol. 2, pp. 1648-1707, hep-th/0311139.

[13] V.M. Braun, S.E. Derkachov, A.N. Manashov, Phys. Rev. Lett. 81 (1998) 2020;

V.M. Braun, S.E. Derkachov, G.P. Korchemsky, A.N. Manashov, Nucl. Phys. B 553 (1999) 355. 
[14] A.V. Belitsky, Phys. Lett. B 453 (1999) 59; Nucl. Phys. B 558 (1999) 259; Nucl. Phys. B $574(2000) 407$.

[15] S.E. Derkachov, G.P. Korchemsky, A.N. Manashov, Nucl. Phys. B 566 (2000) 203.

[16] A.V. Belitsky, V.M. Braun, A.S. Gorsky, G.P. Korchemsky, Int. J. Mod. Phys. A 19 (2004) 4715.

[17] L.A. Takhtajan, L.D. Faddeev, Russ. Math. Survey 34 (1979) 11;

V.E. Korepin, N.M. Bogoliubov, A.G. Izergin, Quantum inverse scattering method and correlation functions, (Cambridge Univ. Press, 1993);

L.D. Faddeev, Int. J. Mod. Phys. A 10 (1995) 1845; Les Houches Lectures (1995), How algebraic Bethe ansatz works for integrable models, hep-th/9605187.

[18] N. Beisert, S. Frolov, M. Staudacher, A.A. Tseytlin, J. High Ener. Phys. 0310 (2003) 037.

[19] R.J. Baxter, Annals Phys. 70 (1972) 193; Exactly Solved Models in Statistical Mechanics, Academic Press (London, 1982).

[20] B. Sutherland, Lect. Notes Phys. 242 (1985) 1; Phys. Rev. Lett. 74 (1995) 816.

[21] G. Mandal, N.V. Suryanarayana, S.R. Wadia, Phys. Lett. B 543 (2002) 81;

I. Bena, J. Polchinski, R. Roiban, Phys. Rev. D 69 (2004) 046002;

A.M. Polyakov, Mod. Phys. Lett. A 19 (2004) 1649.

[22] V.E. Zakharov, A.V. Mikhailov, Sov. Phys. JETP 47 (1978) 1017.

[23] I.M. Krichever, Funct. Anal. Appl. 28 (1994) 21.

[24] V.A. Kazakov, K. Zarembo, J. High Ener. Phys. 0410 (2004) 060.

[25] M. Gaudin, V. Pasquier, J. Phys. A 25 (1992) 5243.

[26] G.P. Korchemsky, Nucl. Phys. B 462 (1996) 333; Nucl. Phys. B 498 (1997) 68.

[27] F.A. Smirnov, Amer. Math. Soc. Trans. 201 (2000) 283.

[28] N. Beisert, Phys. Rept. 407 (2004) 1.

[29] E.K. Sklyanin, Lect. Notes Phys. 226 (1985) 196; Quantum inverse scattering method. Selected topics, "Quantum Group and Quantum Integrable Systems," ed. Mo-Lin Ge, World Scientific, (Singapore, 1992) pp. 63-97, hep-th/9211111; Progr. Theor. Phys. Suppl. 118 (1995) 35.

[30] A.S. Gorsky, Theor. Math. Phys. 142 (2005) 153.

[31] G.P. Korchemsky, Nucl. Phys. B 443 (1995) 255.

[32] S.P. Novikov, S.V. Manakov, L.P. Pitaevsky, V.E. Zakharov, Theory of soliton: the inverse scattering method, Consultants Bureau (New York, 1984).

[33] L.D. Faddeev, L.A. Takhtajan, Hamiltonian methods in the theory of solitons, SpringerVerlag (Berlin, 1987). 
[34] O. Babelon, D. Bernard, M. Talon, Introduction to classical integrable systems, (Cambridge University Press, 2003).

[35] N.Yu. Reshetikhin, F.A. Smirnov, Zap. Nauch. Sem. LOMI 131 (1983) 128.

[36] G.P. Korchemsky, I.M. Krichever, Nucl. Phys. B 505 (1997) 387.

[37] N. Gromov, V. Kazakov, Nucl. Phys. B 736 (2006) 199.

[38] M. Kruczenski, J. High Ener. Phys. 0508 (2005) 014. 